HCl (14:1) oder, noch vollständiger, in konzentrierter Ameisensäure extrahiert. Letztere wurde vor dem Abzentrifugieren mit dem doppelten Volumen Methanol verdünnt. Als bestes Laufmittel bewährte sich das von Butenandt, Biekert und Linzen ${ }^{19}$ angegebene, etwas abgeänderte Gemisch: 85-proz. HCOOH-MethanolHCl konz. im Verhältnis $80: 15: 0,5$. Das rote Pigment konnte außerdem aus ameisensaurer Lösung durch Neutralisieren ausgefällt werden. Die entstehenden roten Flocken wurden mehrmals mit Wasser gewaschen, jedoch der äußerst geringen Menge wegen nicht weiter durch Umfällen gereinigt. Das so erhaltene Produkt zeigte bei chromatographischer Prüfung noch die kräftig blaue Fluoreszenz einer Substanz mit höherem $R_{f}^{6}$ Wert.

Pterorhodin wurde gewonnen durch Mischung salzsaurer Lösungen (in 3-n. $\mathrm{HCl}$ ) von Xanthopterin (Hoffmann-La Roche) und 9-Methylxanthopterin ${ }^{20}$ unter $\mathrm{Zu}$ satz von wenig 15-proz. Lösung von Wasserstoffsuperoxyd $^{8}$. Die bei Zimmertemperatur rasch sich rötlich färbende Lösung wurde zur Trockne eingeengt, mit $\mathrm{HCOOH}$ wieder aufgenommen und $\mathrm{zu}$ papierchromatographischen Vergleichen und für Absorptionsspektren verwendet, die stets unter Verwendung von $\mathrm{HCOOH}$ als Lösungsmittel im Zeiss-Spektralphotometer aufgenommen worden sind.

Aus Extrakten unbelichteter dec-Köpfe konnte rotes Pigment in folgender Weise ${ }^{12}$ hergestellt werden:

19 A. Butenandt, E. Biekert u. B. Linzen, Hoppe-Seyler's Z. physiol. Chem. 312, 227 [1958]. ammoniakalische Fxtrakte aus Alkoholmaterial wurden im Wasserbad eingeengt und auf eine CellulosepulverSäule gegeben. Die intensiv blaugrün fluoreszierende Zone, die außer $c_{1}$ noch Isoxanthopterin und Xanthopterin enthielt, wurde aufgefangen und schonend zur Trockne eingeengt, dann in $n-\mathrm{HCl}$ aufgenommen und bei etwa $80^{\circ} \mathrm{C}$ unter Durchströmung mit Luft erneut eingeengt. Der orangerote Rückstand wurde mit $\mathrm{HCOOH}$ aufgenommen und papierchromatographisch getrennt (HCOOH-Methanol-HCl). Er wurde dabei zerlegt in eine rote Zone, die dem Pterorhodin entsprach, und eine gelbliche mit höherem $R_{f}$-Wert, die wohl noch nicht umgesetztes $c_{1}$ und andere fluoreszierende Pterine enthielt.

Vor den Belichtungsversuchen (S. 656) wurden die Augenfarb- und Helligkeitswerte der dec-Tiere in Äthernarkose mit dem Ostwald-Farbkörper gemessen (Verfahren ${ }^{7}$ ). Dann wurden die Tiere in je einen 100-ml-Erlenmeyerkolben gebracht, von denen der eine mit Stickstoff aus der Stickstoffbombe $(99-99,8 \%$ Reinheit) durchströmt wurde. Der andere Kolben war mit Gaze lose verschlossen und enthielt Luft. Die Belichtung der beiden nebeneinander stehenden Gefäße erfolgte (am Fenster im Sonnenlicht) unter völlig gleichen Bedingungen. Nach Versuchsende wurden die Augen beider Gruppen wieder getrennt mit dem $\mathrm{Ostwald}$ Farbkörper gemessen und dann die Fluoreszenzmuster der Köpfe papierch:omatographisch untersucht.

-0 Für die Überlassung der Substanz danken wir Herrn Professor Tschesche, Hamburg.

\title{
Optokinetische Wirksamkeit bewegter periodischer Muster (Nach Messungen am Rüsselkäfer Chlorophanus viridis)
}

\author{
Von Bernhard Hassenstein \\ Aus dem Max-Planck-Institut für Biologie, Tübingen, Forschungsgruppe Kybernetik \\ (Z. Naturforschg. 14 b, 659-674 [1959] : eingegangen am 18. August 1959)
}

\begin{abstract}
The strength of optomotor reactions has been measured by means of the Y-maze-globe method (Fig. 1). If the moving patterns are composed of vertical stripes of contrasting shades of grey the strength of reaction is a quadratic function of the difference of reflexion between the stripes (Fig. 2). This corresponds to the finding that perception of movement in the beetle's eye results from the multiplication between stimulus intensities of adjacent ommatidia (Fig. 13). Varying velocities of a sinusoidal pattern of shades (Fig. 4) release reactions plotted in Fig. 5. The response to more complicated moving patterns like those shown in Figs. 3, 6, 11, 15 is the sum of the responses to the sinusoidal (F o u rier) components involved. The relative phases of the periodic components of the patterns have no influence on the reaction (Fig. 11, 12). Under special experimental conditions, i. e. high velocity of the moving structures, the physiological response to different shades of grey are linear. In the case of very slow velocities the physiological scale of grey of the beetle's eye is altered by an adaptation process; the medium grey changes its place from grey 50 to grey 40 (experimental black $=0$, white $=100$ ). Moving irregular patterns which are composed of very fine stripes (Fig. 15 b) release reactions plottet in Fig. 16. The reaction curve has a negative part which results from a special mechanism discussed by Reichardt und VARJU ${ }^{4}$. - Most of the above experiments were designed in order to prove theoretical predictions which were derived from the correlation principle of movement perception ${ }^{1,4,5}$.
\end{abstract}

Bei der Spangenglobus-Methode (Abb. 1) beeinflussen die Wendetendenzen des Versuchstieres das Zahlenverhältnis zwischen seinen Rechts- und Linkswahlen.
Diesen Wirkungszusammenhang hielt ich lange Zeit für $\mathrm{zu}$ indirekt und unübersichtlich, um von ihm mathematisch auswertbare Meßwerte für physiologische Seiten- 
tendenzen zu erwarten. Der erste Versuch aber, mit seiner Hilfe eine theoretisch vorausgesagte Reaktionskurve $^{1}$ nachzuprüfen, ergab eine unmittelbare Übereinstimmung zwischen Theorie und Meßwerten ${ }^{2}$. Danach war es wahrscheinlich, daß eine lineare Beziehung zwischen physiologischen Wendetendenzen und Wahlergebnissen am Spangenglobus besteht. Ich suchte nach einer Vorstellung, die einen derart einfachen Zusammenhang verständlich machen könnte; eine plausible Erklärung

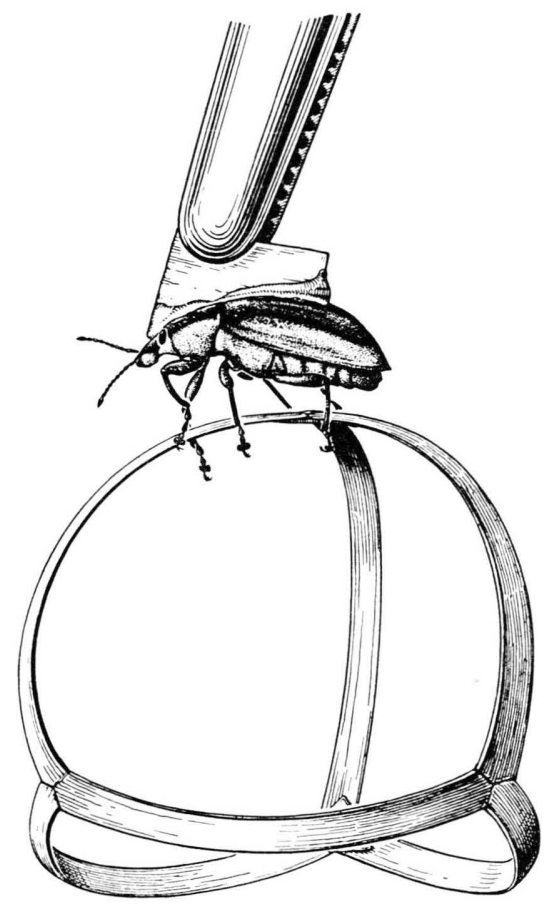

Abb. 1. Das Versuchstier (der Rüsselkäfer Chlorophanus viridis) ist mit seinem Rückenpanzer an ein Stückchen Pappe geklebt, das von einer Pinzette gehalten wird. Der Käfer hängt also zunächst frei in der Luft. In dieser Situation wird ihm ein Gebilde aus Stroh, der „Spangenglobus“ gereicht, den er freiwillig festhält; er ist gerade so schwer wie der Käfer selbst, so daß der Käfer etwas für ihn natürliches tut: Er trägt „sein eigenes Gewicht“, als liefe er mit dem Rücken nach unten an einem Zweig. Der Spangenglobus ist so beschaffen, daß sich seine 6 Spangen an 4 Punkten zu je dreien im Winkel von $120^{\circ}$ zueinander treffen. Macht der Käfer nun Laufbewegungen, so dreht sich der Spangenglobus unter ihm. Nach einigen Schritten kommt der Käfer jedesmal an einen Y-förmigen Scheideweg - oder genauer: Ein solcher Scheideweg kommt auf ihn zu. Er muß sich nun für Rechts oder Links entscheiden. Die Wahlen am Spangenglobus werden in der gleichen Weise durch optische Bewegungsreize beeinflußt wie die Laufrichtung des Tieres auf ebener Fläche. Ohne Bewegungsreiz wählt der Käfer am Spangenglobus gleich häufig „rechts“ und „links“. Einer schwachen Wendung nach rechts entspricht dann z. B. ein Verhältnis von 75 Rechtszu 25 Linkswahlen, einer starken Wendung von 100 Rechtszu 0 Linkswahlen. Im Experiment wird jeder Bewegungsreiz so lange in dauernder Wiederholung gegeben, bis der Käfer häufig genug gewählt hat, um ein statistisch genügendes Wahlverhältnis als Maß für seine jeweilige Wendetendenz zu liefern. Genaueres $1958^{1,3}$ ließ sich finden und ist in der 1958 erschienenen Arbeit ${ }^{2}$ angegeben.

Ich durfte also doch hoffen, daß die SpangenglobusMethode mathematisch diskutierbare Meßwerte liefern könnte. Daher ging ich daran, alle Voraussetzungen und Folgerungen aus Reichardts und Varjús mathematischer Bearbeitung der Bewegungsperzeption von Chlorophanus ${ }^{1,4,5}$, die experimentell zugänglich erschienen, im Versuch nachzuprüfen. Im wesentlichen lief dies auf folgende drei Fragen hinaus: Welche Parameter bestimmen die optokinetische Wirksamkeit bewegter periodischer Muster? Welchem Formalismus folgt die Graustufen-Bewertung im Käferauge? Und: Welcher Art sind die Reaktionen auf die Bewegung eines idealen statistisch unregelmäßigen Helligkeitsmusters? Diese letzte Frage hatte ich schon einmal ${ }^{2}$, aber, wie sich inzwischen ergab, unvollständig bearbeitet.

\section{Versuchstier und Versuchsmethodik}

Die Versuchstiere (Chlorophanus viridis, Rüsselkäfer) wurden in der Umgebung von Wilhelmshaven gesammelt und mit der Post nach Tübingen gesandt.

Die Versuchsmethode war die des „schwebenden Spangenglobus" (Abb. 1), der Versuchsaufbau der gleiche wie $1958^{2}$ beschrieben. Der Quotient aus Mitund Gegenwahlen zur Musterbewegung, der am Spangenglobus die Reaktionsstärke angibt, nähert sich bei starken Seitentendenzen den Werten +1 bzw. -1 ; er kann, wenn er sie erreicht hat, auch bei noch weiter wachsender Seitentendenz nicht mehr weiter zunehmen $(+1$ heißt, daß bereits sämtliche gezählten Wahlen Mitwahlen wahren). Dieser Extremfall offenbart also eine starke Nichtlinearität der Meßmethodik. Die oben erwähnte Linearität kann daher nicht über die gesamte Meßskala gültig sein. Erfahrung und theoretische Überlegung ${ }^{2}$ stimmen darin überein, daß sich der bei unserer Meßgenauigkeit praktisch lineare Bereich bis zum Wert $\pm 0,7$ erstreckt.

Um dies zu berücksichtigen, wurde vor Beginn jedes Versuchs diejenige Seitentendenz ausgelöst, die nach den bisherigen Erfahrungen und Deutungen im folgenden Versuch die Maximal-Reaktion darstellen mußte. Ergaben sich dabei Werte $0,5<R<0,7$, so lag der Messung nichts mehr im Wege. Lag der Meßwert aber zu niedrig oder zu hoch, so veränderte ich einen derjenigen Versuchsparameter, deren Einfluß auf die zu untersuchende Reaktion vermutlich ohne Belang war. Meist war dies die Schlitzbreite des Rasterzylinders. Mit ihr änderte ich die Amplitude der Helligkeitswechsel für die einzelnen Sehelemente, und zwar geschah dies im Lauf der Untersuchungen in einem Bereich von $1: 3$, was wegen der multiplikativen Verrechnung einem Reaktionsstärken-Bereich von 1 : 9 entspricht. Durch Halbierung der Schlitz-Höhe schwächte ich einige Male die Reaktion um einen weiteren Faktor 2 ab. Umgekehrt brauchte ich in Versuch 10 eine zusätzliche Verstärkung

1 W. Reichardt, Z. Naturforschg. 11 b, 448 [1957].

2 B. Hassenstein, Z. Naturforschg. 12 b, 1 [1958]. 
der Reaktion: Durch Verdoppelung der Schlitz-Anzahl geschah dies mit dem Faktor 4. - Erst nachdem ich auf solche Weise einen geeigneten Maximal-Meßwert für den kommenden Versuch gewonnen hatte, behielt ich die gewählte Anordnung bei und führte die Messungen aus.

Die Norm-Anzahl der gezählten Wahlen pro Einzelmessung erhöhte ich auf 26. Zur Mittelwertbildung verwertete ich nicht mehr wie früher unbedingt alle angestellten Messungen, sondern schied einige Male solche Meßkurven aus, die eine übermäßig starke Streuung zeigten, also z. B. in einem mit Sicherheit monotonen Reaktionsanstieg eine ein- oder mehrfache Richtungsumkehr zeigten. Wieviele Kurven in diesem Sinne bei der Schlußauswertung weggelassen wurden, wird jeweils bei den Einzelversuchen angemerkt.

In fast allen Versuchen war der Käfer von einem Rasterzylinder umgeben, dessen $1,7^{\circ}$ breite Schlitze (Fenster) $14^{\circ}$ voneinander entfernt waren. So wurde durch die Drehung der Zylinder stets nur jedes zweite Ommatidium gereizt. Damit war gewährleistet, daß für alle Einzelreaktionen, die das experimentelle Ergebnis zusammensetzten, der gleiche Winkelabstand zwischen den Eingangselementen $\left(14^{\circ}\right)$ maßgebend war.

Die bewegten Muster enthielten oft verschieden helle Grauwerte. Diese wurden durch Mischung aus „Plaka-Farben“ Schwarz und Weiß gewonnen. Die Mischungen wurden mit wenig Wasser verdünnt, mit einem breiten Pinsel auf Chromatographiepapier aufgetragen und photometriert. Die Reflexion des mit reiner weißer Plakafarbe erreichbaren "Weiß" wird im folgenden gleich 100 oder, wo dies zu bequemeren Formulierungen führt, gleich 1 gesetzt. Plaka-,,Schwarz“ reflektiert in den dadurch definierten Einheiten mit der Intensität 4, schwarzer Samt mit der Intensität 2. Als „mittleres Grau“ wird im folgenden dasjenige Grau bezeichnet, das mit der Intensität 52 reflektiert, also das arithmetische Mittel zwischen „Schwarz“ und „Weiß“ bildet. Für das menschliche Auge liegt dieses Grau viel näher dem Weiß als dem Schwarz; „Mittelgrau" für das menschliche Auge war nach den Schätzungen von 10 Personen Grau 24 (Extremwerte: Grau 31 und 17).

Als „Kontrast“ wird im folgenden der absolute Wert des Helligkeitsunterschieds zwischen scharf aneinandergrenzenden Flächen eines Musters bezeichnet. Die Kontaktlinie zwischen verschieden hellen Flächen ist eine „Kontur“. Bewegt sich eine Kontur über das Gesichtsfeld eines Sehelements, so erfolgt dort ein „Helligkeitssprung". Je mehr Konturen pro Längeneinheit, desto mehr Helligkeitssprünge erfolgen pro Zeiteinheit für das Insektenauge bei gleichschnell bewegten Mustern.

Die Helligkeit der weißen Musteranteile der Zylinder um das Versuchstier war bei der normalen VersuchsBeleuchtung, gemessen mit einem Luxmeter, gleich $0,03 \%$ der Sonnenintensität oder weniger. Da Luxmeter nicht auf Lampenlicht, sondern auf Licht mit der spektralen Zusammensetzung des Sonnenlichtes geeicht sind, lassen sich hieraus Schlüsse auf die Reizenergie nur mit einem Unsicherheits-Faktor von 2 bis 3 ziehen. Wieviel Lichtenergie durch die Schlitze der Rasterzylinder in die einzelnen Ommatidien fiel und dort die Reizwirkung entfaltete, ist schwer abzuschätzen; denn wahrscheinlich wird ein kleiner Lichtfleck je nach seiner Lage zur Ommatidien-Mittelachse sehr verschieden stark rezipiert ${ }^{5}$. Der von der Musterhelligkeit getroffene Anteil der Gesichtsfelder der Einzelommatidien wird durch die Rasterzylinder im ganzen auf 50 bis $10 \%$ eingeschränkt.

\section{Versuche}

\section{Variation der Kontraste}

Die Bewegung eines Musters ist prinzipiell nur an Stellen der Helligkeitsänderung, nicht aber an Flächen gleichmäßiger Tönung zu erkennen. Die Wirkungseinheiten bewegter Muster für ein wahrnehmendes Auge könnten danach im Falle scharf abgesetzter Musteranteile allein die Konturen sein. Um darum die Konturenwirkung näher kennenzulernen, soll der erste Versuch klären, wie die Größe der Hell-Dunkel-Kontraste an den Konturen eines Streifenmusters die Stärke der optokinetischen Wendetendenz von Chlorophanus beeinflußt.

Die Versuchstiere wurden mit dem Spangenglobus im Zentrum des beschriebenen $14^{\circ}$-Rasterzylinders fixiert; außen rotierte der von einem Elektromotor angetriebene Zylinder, der die Helligkeitsmuster trug. Seine Winkelgeschwindigkeit blieb während jeder Meßreihe konstant; sie wurde vor Beginn des Versuches so eingestellt, daß die Reaktionsintensität des jeweiligen Versuchstieres bei Verwendung des am stärksten wirksamen Zylinders (in allen Fällen beim Schwarz-Weiß-Kontrast) etwa $+0,7$ betrug.

Veränderte Helligkeitskontraste innerhalb der bewegten Muster wurden auf drei Weisen hervorgebracht. Den Ausgang bildete stets ein Muster aus schwarzen und weißen Streifen. In der ersten Meßreihe wurde der Kontrast stufenweise dadurch verändert, daß sowohl die schwarzen als auch die weißen Streifen durch Graustreifen ersetzt wurden, die sich von Mal zu Mal um etwa gleichgroße Schritte gegen „Mittelgrau“ hin veränderten. Auf diese Weise blieb die Durchschnittshelligkeit nahezu gleich und entsprach etwa dem mittleren Grau. Die gegeneinander kontrastierenden Streifen in diesen Mustern waren gleich breit. - In den beiden anderen Meßreihen wurde entweder das Weiß (zweite Meßreihe) oder das Schwarz (dritte Meßreihe) festgehalten und nur die dagegen kontrastierenden Grautöne abgewandelt. Hierbei waren stets die weißen bzw. schwarzen Streifen schmaler als der graue Musteranteil. In der zweiten und dritten Meßreihe änderte sich also außer dem Kontrast auch die mittlere Helligkeit der Muster*.

\footnotetext{
* Die mittlere Abweichung $\sigma$ der Helligkeitswerte $L$ der Muster vom Helligkeits-Mittelwert $C$, h. h. $\sigma=|C-L|$, ändert sich in allen drei Anordnungen proportional dem Kontrast. Dies ist von Bedeutung im Hinblick auf Versuch 4.
} 


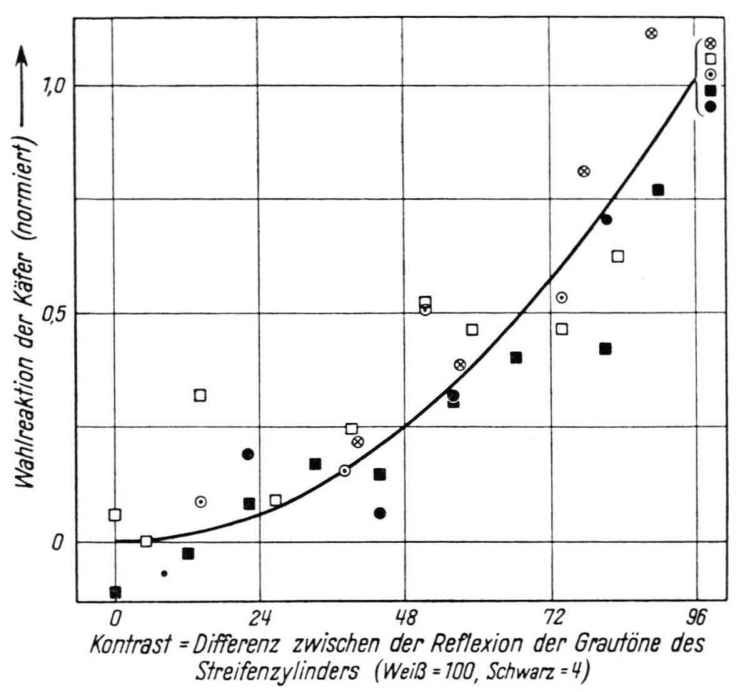

Abb. 2. Reaktion auf bewegte Muster in Abhängigkeit vom Kontrast zwischen den Musterbestandteilen. $\otimes$ : Erste Meßreihe (s. Text); Durchschnittsergebnisse aus 5 Versuchen. $\square$ und $\odot$ : Zweite Meßreihe; Durchschnitt aus je 3 Versuchen. und : Dritte Meßreihe; Durchschnitt aus je 3 Versuchen. Die Winkelgeschwindigkeit des Musters war von Versuch zu Versuch verschieden, um die jeweils günstigste Maximal-Reaktionsstärke einzustellen, und lag zwischen $10^{\circ} / \mathrm{sec}$ und $560 \%$ sec. Von der Mustergeschwindigkeit abhängige Unterschiede im Reaktionsverlauf waren nicht festzustellen bzw. lagen unter meiner Meßgenauigkeit.

Abb. 2 zeigt die Stärke der optokinetischen Wendetendenzen der Versuchstiere in Abhängigkeit von den Kontraststärken in den bewegten Mustern. In allen Fällen wurde die Maximalreaktion der Einzelmessungen für die Auftragung gleich +1 gesetzt. Wie man sieht, ergaben die Ergebnisse der drei Meßreihen im Rahmen der erreichten Genauigkeit keine Unterschiede gegeneinander. Wie man weiter erkennt, scharen sich die Meßpunkte nicht um die Gerade, die den Punkt \pm 0 mit dem der Maximalreaktion verbindet, und erst recht nicht um eine logarithmische Charakteristik, wie sie der Helligkeitsempfindung des menschlichen Auges, bezogen auf die arithmetische (,physikalische“) Graureihe, entspricht. Vielmehr gruppieren sich die Meßpunkte, soweit dies bei der Streuung der Ergebnisse deutlich wird, um die in Abb. 2 eingezeichnete quadratische Kurve.

Die Streuung der Meßpunkte ist allerdings ziemlich groß. Das liegt an der für die SpangenglobusMethode ungünstigen Form und Lage der Reaktionskurve: Sie bewegt sich auf mehr als der Hälfte ihres Verlaufs nahe der Null-Linie und besitzt keine scharf abgehobene Formmerkmale wie Nulldurchgänge oder Maxima und Minima. Das Versuchsergebnis Abb. 2 zeigt daher wohl die in der Sinnesphysiologie ungewohnte nach oben konkave Reaktionskurve auf wachsende Reizintensitäten, läßt aber noch andere Zusammenhänge als den quadratischen zu.

Nach den Ergebnissen der Versuche 8 und 9 (S. 669,670 ) ist jedoch an der im Prinzip quadratischen Reaktionscharakteristik nicht zu zweifeln. Wie sich dort zeigt, entspricht die physiologische Grauskala des Käferauges bei den hier verwendeten ZylinderGeschwindigkeiten und Rasterzylindern genau der arithmetischen Graureihe (Verzerrung unter den hier vorliegenden Bedingungen nicht größer als $5 \%$ ) ferner wirken die linear übersetzten Reizgrößen je zweier benachbarter Sehelemente multiplikativ zusammen. Da nun in diesem Versuch jeweils gleichartige Reize nacheinander in benachbarte Ommatidien fallen, muß sich durch die lineare Übersetzung und die nachfolgende Multiplikation eine quadratische Abhängigkeit von der Kontraststärke ergeben.

\section{Verdopplung der Konturen-Anzahl bei Halbierung der Kontraste}

Wenn es die Konturen sind, die die optokinetische Wirksamkeit eines Musters bestimmen, so könnte außer ihrer Kontrasthöhe (Versuch 1) auch ihre Anzahl pro Musterabschnitt, also der Konturenreichtum des Musters, von Einfluß sein. Um dies ohne Veränderung der Periodenlänge des Musters zu testen, wurde im folgenden Versuch zunächst ein SchwarzWeiß-Muster geboten, dann der Kontrast an jeder Einzelkontur halbiert und die Anzahl der Konturen verdoppelt (Abb. 3). Da die Wirkung jeder Einzelkontur damit gemäß Versuch 1 auf ein Viertel ihres früheren Wertes absank, mußte sich am Versuchsergebnis erkennen lassen, mit welchem Faktor die Konturenvermehrung in das Ergebnis einging. In früheren Versuchen ${ }^{3}$ hatten sich stets alle gleichzeitig vom Käferauge wahrgenommenen Einzelbewegungen in ihrer Wirkung auf die entstehende Seitentendenz summiert; ich vermutete daher, auch bei der Vervielfachung der Reize in der Zeit würden sich die Einzelwirkungen einfach überlagern. War dies richtig, so mußte die zweifache Konturen-Vermehrung fähig sein, die Auswirkung der Halbierung der Kontrastgrößen gerade zur Hälfte wettzumachen. Ich prüfte die beiden Muster: Schwarz-Weiß und Schwarz-Grau-Weiß-Grau gleicher Periodenlänge $\left(90^{\circ}\right)$, bei 9 Winkelgeschwindigkeiten zwischen 6,9

3 B. Hassenstein, Z. vergleich. Physiol. 40, 556 [1958]. 
und $560^{\circ} / \mathrm{sec}$ und erhielt dadurch zwei Kurven der Reaktionsstärke in Abhängigkeit von der Mustergeschwindigkeit. Wie Abb. 3 zeigt, sind sie tatsächlich um etwa den erwarteten Faktor 2 voneinander verschieden. In erster Näherung schien damit die obige Voraussage bestätigt. Über die Kurvenform und die Lage des Maximums wird bei Versuch 5 und 6 gesprochen werden.

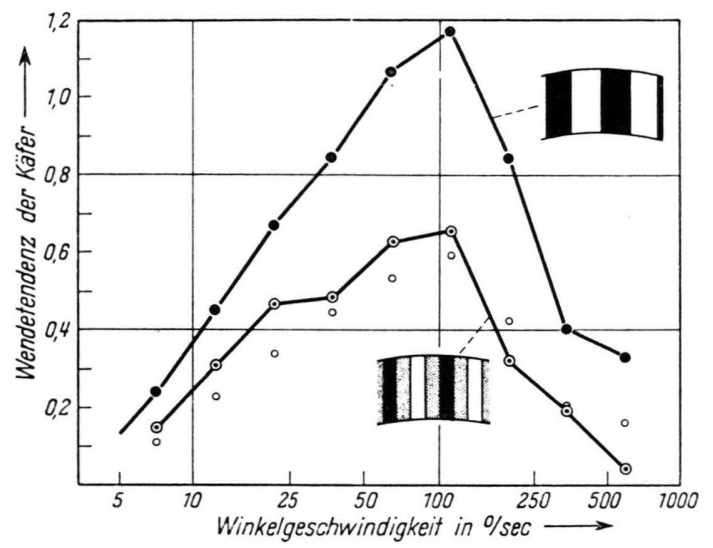

Abb. 3. Reaktion auf die Bewegung zweier periodischer Muster mit der Periodenlänge $90^{\circ}$. Durchschnittsergebnisse von zwei Versuchstieren; 3 Meßkurven mit zu großer Streuung nicht verwertet. Die kleinen offenen Kreise geben die halben Ordinatenwerte der oberen Kurve an, um den Vergleich mit der unteren Kurve zu erleichtern. Die Wendetendenzen $R$ wurden nach der Formel $W(R)=2 \Phi(1,25 R)$ aus den registrierten Spangenglobus-Wahlreaktionen $W(R)$ errechnet $(\Phi$ ist das Integral der G a u $\beta$ schen Zufallsverteilung)* .

\section{Muster mit sinusförmig variierender Helligkeit}

Beim vorigen Versuch hatte die Vorstellung, daß Kontrastgrößen quadratisch und Konturenhäufigkeiten proportional in die Reaktionsstärke eingingen, zu einer zutreffenden Voraussage geführt. Was war aber zu erwarten, wenn der Helligkeitsumfang zwischen den verwendeten Grenzwerten Schwarz und Weiß in immer kleinere Schritte aufgeteilt wurde? Eine Helligkeitsverteilung mit fließenden Übergängen, z. B. ein sinusförmig variiertes Helligkeitsmuster dürfte dann, falls die Vorstellung richtig war, durch seine Bewegung gar keine optokinetischen Reaktionen mehr auslösen.

Das Muster, das den Käfern in Bewegung gezeigt wurde, war nur eine Annäherung an eine sinusförmige

* Korrektur: In der Arbeit von $1958^{2}$, in der diese Formel angegeben wurde, stand statt des Faktors 1,25 versehentlich der Faktor 2,5.
Helligkeitsverteilung (Abb. 4). Es war aus schmalen Streifen von 10 geeichten Grauwerten zusammengesetzt; die Reaktionsstärke hätte somit im Vergleich zum Schwarz-Weiß-Zylinder mindestens um eine Größenordnung, also auf ein Zehntel $\left(10^{-2} \cdot 10^{1}\right)$ absinken müssen. Wiederum wurde eine Kurve der Geschwindigkeits-Abhängigkeit der Reaktion aufgenommen; die Winkelgeschwindigkeit der Muster variierte in 10 Schritten zwischen 1,1 und $1156^{\circ} / \mathrm{sec}$.
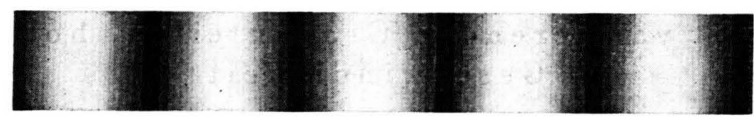

Abb. 4. Annähernd sinusförmiges Schwärzungsmuster, her gestellt durch Nebeneinandersetzen von Graupapierstreifen bekannter Helligkeit.

Abb. 5 zeigt das Versuchsergebnis. Das Erwartete trat nicht ein: Die Reaktionsstärke lag im gleichen Bereich wie im vorigen Versuch (genaue Messung in Versuch 5). - Die Geschwindigkeits-Abhängigkeit, bei logarithmischer Auftragung der Geschwindigkeit, stellt eine glockenförmige Kurve dar. Ihr Anstieg und Abstieg sind recht genau symmetrisch zueinander. Das Maximum liegt bei $50 \%$ sec. Am Maximum bildet die Kurve keine scharfe Spitze wie die Kurven der Abb. 3, sondern ist breit abgerundet. Die Halbwertsbreite der Kurve (hier ausgedrückt durch den Quotienten der Abszissenwerte beim halben Maximalwert der Reaktion) ist rund 14.

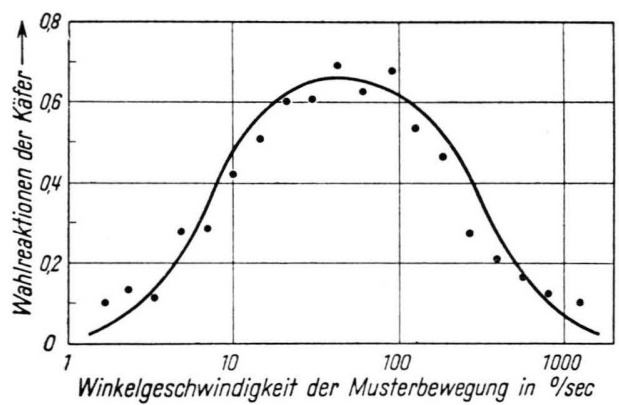

Abb. 5. Reaktionen auf die Bewegung des angenäherten sinusförmigen Helligkeitsmusters Abb. 4; Periodenlänge $66^{\circ}$. Durchschnittswerte von 4 Meßkurven; eine Messung wegen zu großer Streuung nicht verwertet. Theoretische Kurve nach REICHARDT und VarJú ${ }^{4}$.

Die Reaktionen der Abb. 5 sind von Reichardt und VARJú zur Grundlage einer mathematischen Bearbeitung der Bewegungsperzeption von Chlorophanus gemacht worden ${ }^{4,5}$. Sie konnte viel weiter ins einzelne gehen als die erste Bearbeitung ${ }^{1}$, da die

4 W. Reichardt u. D. Varjú, Z. Naturforschg. 14 b 674 [1959].

5 D. VARJu', Z. Naturforschg. 13 b, [1959], im Druck. 
im Experiment verwendete Sinus-Verteilung ihrer in die Überlegungen eingesetzten mathematischen Formulierung viel besser entsprach, als dies bei statistisch unregelmäßigen Mustern möglich ist. Dies wird an Hand der Ergebnisse des Versuches 10 (S. 672) näher besprochen werden.

\section{Schwarz-Grau-Wei $ß-G$ rau-Muster} mit variierenden Graunteilen, hohe Geschwindigkeit

Da die Erklärung des Ergebnisses von Versuch 2 (Abb. 3) zu einer falschen Voraussage über die optokinetische Wirksamkeit des Sinusmusters geführt hatte, lag der Verdacht nahe, daß diese erste Erklärung falsch war. Ihr Grundgedanke war, daß sich für das Käferauge die Wirkung eines bewegten Musters summativ aus den Wirkungen der bewegten Einzelkonturen zusammensetzt. Um diese Vorstellung nochmals unmittelbar zu prüfen, bot ich dem Käferauge die auf Abb. 6 skizzierten Muster aus Schwarz- und Weiß-Anteilen mit dazwischen eingeschobenen GrauFlächen. Sie enthalten - bis auf den ersten, zum Vergleich gebotenen reinen Schwarz-Weiß-Zylinder - gleichviele Konturen von gleichem Kontrastwert. Was von einem Muster zum nächsten variiert, ist die relative Flächengröße der verschiedenen Anteile, also ein Parameter, der nach der vorigen Anschauung für das Bewegungssehen belanglos sein müßte. Die gebotenen Schwarz-Grau-Weiß-Grau-Muster hätten also gleich wirksam sein müssen.

D. VARJù riet mir zu Streifenzylindern mit der Periodenlänge $360^{\circ}$ (also pro Zylinder nur ein Schwarz- und ein Weißanteil), und zu der hohen Mustergeschwindigkeit von $950^{\circ} / \mathrm{sec}$. Ich verwendete 8 Muster, bei denen die Graustreifenbreite $0^{\circ}$ bis $164^{\circ}$ betrug. $180^{\circ}$ Grauanteil wäre gleichbedeutend mit einem konturlosen grauen Zylinder ohne Schwarz-Weiß-Anteil gewesen.

Abb. 6 zeigt die Wahlreaktionen der Käfer. Die Meßpunkte liegen durchaus nicht bei gleichen Reaktionsstärken, sondern bilden annähernd eine Gerade, die die gemessene Reaktion auf den Schwarz-WeißZylinder mit der Reaktion Null auf den konturlosen Zylinder verbindet. Daraus folgt: Die Konturen sind für das Käferauge nicht die alleinigen Wirkungsträger in bewegten Mustern.

Auf der nun erneut notwendig gewordenen Suche nach den wirksamen Parametern der Muster muß man feststellen, welche Mustereigenschaften auf Abb. 6 proportional zu den Reaktionsstärken der Käfer variieren. Ein Blick auf die Abbildung läßt erkennen, daß die Breite der Schwarz- und Weiß- flächen diese Forderung im Rahmen der Meßgenauigkeit erfüllt; sie ist dem Meßergebnis annähernd proportional. Dieses nicht zu bezweifelnde experimentelle Ergebnis widerspricht anscheinend dem Grundsatz, daß gleichmäßig getönte Flächen keine Wirkungsträger optischer Bewegungen sein können (s. Einleitung zu Versuch 1); nach ihm hätte es keine

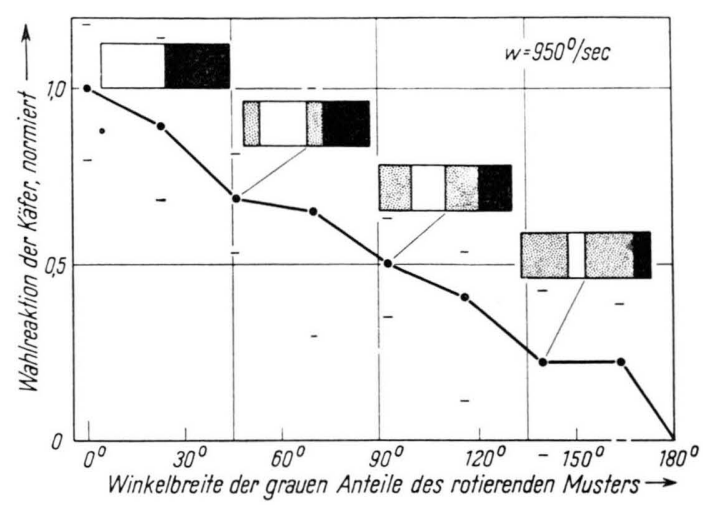

Abb. 6. Reaktionen auf die Bewegung von Schwarz-GrauWeiß-Grau-Mustern. Periodenlänge $360^{\circ}$. Variable: Die Breite des grauen Anteils. Winkelgeschwindigkeit $950 \%$ sec. Mittelwerte aus sämtlichen angestellten Messungen. Extreme Meßwerte durch waagerechte Striche angedeutet. Sie geben die größten vorgekommenen Abweichungen nach Normierung der einzelnen Meßkurven auf den Durchschnittswert der Mittelwert-Kurve an. (Normierung: Errechnung des Durchschnittswertes der Kurvenpunkte der Mittelwertskurve $D_{\mathrm{m}}$ sowie jeder Einzelkurve $D_{\mathrm{n}}$. Multiplikation jedes einzelnen Kurvenpunktes mit dem für seine Reaktionskurve gültigen Wert $\left.D_{\mathrm{m}} / D_{\mathrm{n}}\right)$.

Rolle spielen dürfen, in welchen Verhältnissen die Helligkeiten weiß, grau und schwarz auf die vorhandenen Flächen verteilt sind, da diese als solche ja wirkungslos sein sollten. Die Bedingung, daß die Konturen ihre Eigenschaften unverändert beibehielten, blieb ja während des Versuchs (Abb. 6) erfüllt.

Trotz dieser Schwierigkeit, die sich erst durch Versuch 7 aufklären wird, soll obige formale Feststellung so weit verfolgt werden wie möglich: In diesem Versuch (Abb. 6) wurden die Anteile der verschiedenen Musterhelligkeiten variiert und eine Proportionalität der Reaktion zum Vorkommen (Häufigkeit) der vom Durchschnittswert „Mittelgrau" abweichenden Helligkeiten gefunden. In Versuch 1 (Abb. 2) war - wenn man nun auch bei ihm nicht mehr die Kontraste, sondern die Abweichungen vom Mittelwert der Musterhelligkeiten betrachtet - die Häufigkeit der abweichenden Helligkeiten konstant gelassen, aber der Betrag (Helligkeitsintervall) der Abweichungen variiert worden; 
dieser ging quadratisch in die Reaktionsstärke ein. Beide Versuche zusammen genommen ergaben also: Die Stärke der Reaktion hängt proportional von der Häufigkeit und quadratisch vom Betrag der Helligkeits-Abweichungen vom Mittelwert ab. Diesem zwiefältigen experimentellen Tatbestand korrespondiert nun ein in der mathematischen Statistik verwendeter Begriff, der sich dort aus ganz andersartigen Erwägungen herausgebildet hat: Der Begriff der mittleren quadratischen Abweichung vom Mittelwert einer Verteilung (eigentlich: Mittel aus den Quadraten der Einzelabweichungen). Mit seiner Hilfe läßt sich das kombinierte experimentelle Ergebnis folgendermaßen formulieren: Die Reaktion verhält sich proportional der mittleren (Versuch 4) quadratischen (Versuch 1) Streuung der Helligkeiten des bewegten Musters. - Diese Formulierung soll nun als Leitfaden für die nächsten Versuche dienen.

\section{Schwarz-Grau-W ei $\beta-G$ rau-Muster und Sinusmuster}

Die erste Vorstellung, daß sich die optokinetische Musterwirkung aus Konturenwirkungen zusammensetzt, hatte sich bei der Reaktion auf ein bewegtes Sinusmuster (Versuch 3, Abb. 4) nicht bestätigt. Wie verhält es sich in dieser Hinsicht mit der soeben aus Versuch 4 hergeleiteten Formulierung? Vergleicht man ein sinusförmiges Helligkeitsmuster mit einem Schwarz-Weiß-Muster gleicher Periodenlänge auf die mittlere quadratische Abweichung der Musterhelligkeiten vom Helligkeits-Mittelwert, so liefert die Berechnung ein Verhältnis von genau $1: 2$. Setzt man dies in Beziehung zu Versuchsergebnis Abb. 6, so ergibt sich: Nach der oben entwickelten Vorstellung müßte die Wirksamkeit eines Sinusmusters mit der Wirksamkeit eines Schwarz-Grau-Weiß-Grau-Musters übereinstimmen, dessen Graustreifen gerade $90^{\circ}$, d. h. ebenso breit sind wie die Schwarz- und die Weiß-Streifen.

Auch dieser Versuch wurde als GesçwindigkeitsTest durchgeführt; Ein Sinus- und ein Schwarz-GrauWeiß-Grau-Muster der Periodenlänge $72^{\circ}$ wurden bei 11 verschiedenen Geschwindigkeiten zwischen 2,8 und $670^{\circ} /$ sec geprüft. Wie Abb. 7 zeigt, liegen beide Reaktionskurven außerordentlich nahe beieinander; die eben getroffene Voraussage über die Reaktionsstärke hat sich also bewährt.

Zwischen den beiden Kurven bestehen geringe FormUnterschiede: Sie entsprechen denen, die beim Vergleich der Reaktionskurven von Abb. 3 und 5 auffallen. Auch dort ist die Reaktionskurve auf scharf konturierte Streifenmuster am Maximum spitzer als die auf Sinusmuster; die Spitze liegt etwas unsymmetrisch zwischen An- und Abstieg, und die Halbwertsbreite ist etwas geringer (12 gegen 14). Da diese Unterschiede in beiden, unabhängig voneinander ausgeführten Meßreihen in gleicher Weise hervortreten, vermute ich, daß sie reell sind. Bei der Biene sind sämtliche Reaktionskurven auf Geschwindigkeits-Variation so spitz wie auf Abb. 3, auch beim Darbieten von Sinusmustern (vgl. Abb. 9). Eine theoretische Erklärung für dieses Phänomen besitzen wir noch nicht.

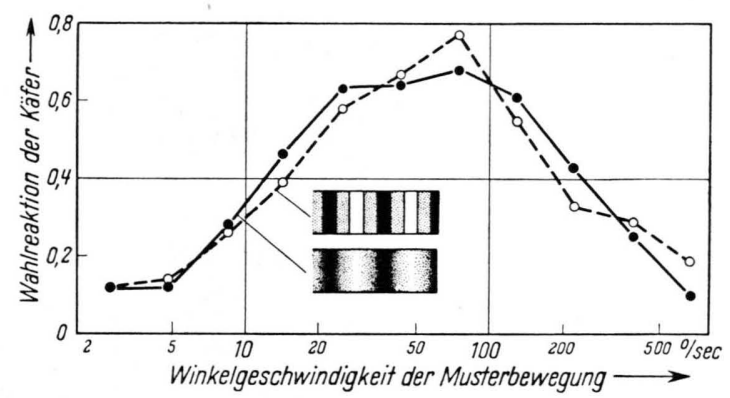

Abb. 7. Reaktionen auf die Bewegung eines Schwarz-GrauWeiß-Grau-Musters und des angenähert sinusförmigen Helligkeitsmusters Abb. 4; Periodenlänge jetzt (bei gegen Abb. 5 etwas verkleinertem Zylinder-Radius) $72^{\circ}$. Beide Kurven wurden mit 2 verschiedenen Käfern aufgenommen; jedesmal wurden beide Muster in einem fortlaufenden Experiment mit demselben Käfer geprüft. Die Abbildung zeigt die Durchschnittswerte aus den beiden angestellten Messungen.

\section{Schwarz-Grau-W ei $\beta-G$ rau-Muster mit wechselndem Grauanteil, geringe Geschwindigkeit}

Bei der mathematischen Bearbeitung der experimentellen Ergebnisse fand VarJú, daß auch die eben erarbeitete Vorstellung, die mittlere quadratische Helligkeitsabweichung vom Mittelwert bestimme die Reaktionsstärke, nicht allgemeingültig sein kann; er riet mir, um dies nachzuprüfen, den Versuch 4 (Schwarz-Weiß-Zylinder mit wechselnden Grauanteilen) bei der Winkelgeschwindigkeit $35^{\circ} /$ sec zu wiederholen. In der Tat ergab sich bei dieser um den Faktor 27 geringeren Geschwindigkeit eine ganz andere Reaktionskurve (vgl. Abb. 8 mit Abb. 6) :

Die Meßpunkte bilden nicht mehr eine schräg verlaufende gerade Linie. Fast alle Zylinder mit Grauanteil lösten jetzt etwa gleichstarke Reaktionen aus; diese lagen beim halben Wert der Schwarz-WeißZylinder-Reaktion. Nur bei den schmalsten unter den verwendeten Graustreifen war die Reaktion geringer.

Es gibt eine anschauliche Deutung für den Unterschied zwischen den Kurven der Abb. 8 und Abb. 6. Bei gerin- 
ger Geschwindigkeit sind die Pausen zwischen den Wirkungen der einzelnen Konturen länger als bei hohen. Bei unendlich langen Pausen müßte die Gesamtreaktion die Summe der Wirkungen der einzelnen Konturen darstellen. In diesem nicht realisierbaren Extremfall müßten nach Versuch 2 alle Muster mit Grauanteil eine genau halb so starke Reaktion auslösen wie Schwarz-WeißMuster gleicher Periodenlänge ohne Grauanteil. Es ist plausibel, daß dieser Zustand bei geringeren Mustergeschwindigkeiten besser angenähert wird als bei höheren; gerade dies ist die Aussage der Kurven Abb. 8 und Abb. 6. - Betrachten wir nun die Kurve Abb. 6 im Lichte dieser Vorstellung und vergleichen sie mit Abb. 8, so müssen wir schließen: Ihre abweichende Form muß auf einer Wechselwirkung zwischen aufeinanderfolgenden Einzelauswertungen beruhen, die bei größerem Zeit. abstand zwischen den Einzelreizen kleiner wird.

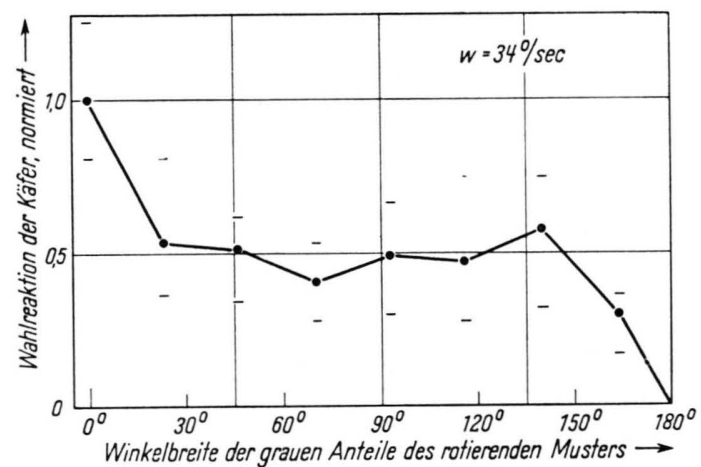

Abb. 8. Derselbe Versuch wie Abb. 6, aber bei der Winkelgeschwindigkeit $34^{\circ} /$ sec. Mittelwerte aus 5 Meßkurven. Eine Kurve wegen $\mathrm{zu}$ starker Streuung nicht verwertet. Extreme Meßwerte wie bei Abb. 6 von normierten Einzelkurven.

Weder die Summe der Konturenwirkungen noch der durchschnittliche Umfang der Helligkeitsvariation können nunmehr die entscheidenden Parameter für die optokinetische Wirksamkeit eines periodischen Musters sein. Hier führte eine theoretische Überlegung weiter: Es glückte VARJú, zwei Parameter zu finden, die die Versuchsergebnisse 1 bis 6 widerspruchslos zu beschreiben gestatten: Praktisch jede periodische Funktion läßt sich nach Fourier in eine Summe von Sinusfunktionen zerlegen. Legt man die Reaktion der Käfer auf eine einzige Sinuskomponente zugrunde (Abb. 5), so erweisen sich die Reaktionskurven Abb. 6 und 8 als Summen aus den Reaktionskurven auf die einzelnen im Muster enthaltenen F o urier-Komponenten; dabei muß die Amplitude der einzelnen F o u ri e r - Komponenten gemäß Versuch 1 quadratisch berücksichtigt werden, und ihre Periodenlänge nach einem Formalismus, der mathematisch aus dem Korrelationsprinzip ${ }^{1}$ hervorgeht, und der experimentell zuerst von Kunze an Bienen ermittelt wurde ${ }^{6}$ (s. folgender Abschnitt).

6 a. Gleichartige Muster verschiedener Periodenlänge

Bietet man dem Auge der Biene oder des Rüsselkäfers gleichartige periodische Muster verschiedener Wellenlänge bei variierender Geschwindigkeit, so entstehen Reaktionskurven gleicher Form; verschieden sind sie jedoch in ihrer Amplitude und in ihrer Lage auf der Geschwindigkeitsachse: Sie verschieben sich bei wachsender Periodenlänge zu größeren Geschwindigkeiten. Die Verschiebung ist der Wellenlängen-Veränderung proportional.

Diesen Tatbestand habe ich bei Chlorophanus nicht isoliert untersucht; doch kommt er bei der Überlagerung periodischer Muster verschiedener Perigodenlängen (Versuch 7) zum Ausdruck. Abb. 9 zeigt Meßergebnisse von Kunze an Bienen ${ }^{6}$. Sie offenbaren die Verschiebung an isoliert gemessenen Kurven. Die Amplituden-Veränderung bezieht sich bei Wellenlängen, die in der Nähe der Rasterkonstante des Auges oder des verwendeten Rasterzylinders liegen, nicht nur auf den Betrag, sondern auch auf das Vorzeichen (Scheinbewegung auf Grund „geometrischer Interferenzen"; s. Abb. 10).

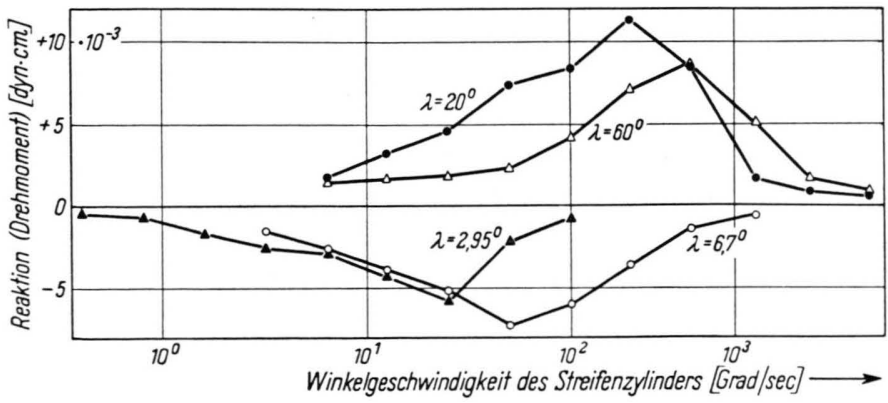

6 P. Kunze, Z. vergleich. Physiol., in Vorbereitung.
Abb. 9. Reaktion von Bienen auf verschieden schnelle Rotation von Streifenmustern verschiedener Periodenlänge $\lambda$. Je kleiner $\lambda$, bei desto kleineren Winkelgeschwindigkeiten liegt das Maximum der Reaktion. Abstand der Schlitze im Rasterzylinder $5^{\circ}$. Messungen von P. Kunze, Z. vergleich. Physiol., in Vorbereitung. 


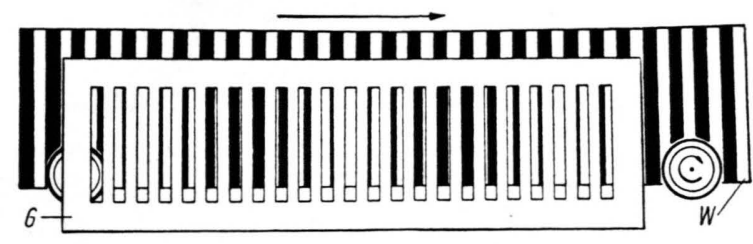

Abb. 10. Zur Veranschaulichung der Entstehung geometrischer Interferenz-Bewegungen. Wenn sich die Streifenwand W nach rechts bewegt, erscheint durch das Raster $G$ eine Scheinbewegung nach links. Man gehe von der Beobachtung aus: Was geschieht in dem Rasterfenster links von demjenigen Fenster, das gerade ganz schwarz ist, wenn sich die Streifenwand nach rechts bewegt?

7. Durch die Überlagerung mehrerer periodischer Komponenten entstandene Muster

Die mit allen bisherigen Ergebnissen im Einklang stehende These, daß sich die optokinetische Wirksamkeit eines Musters für das Käferauge aus den Einzelwirkungen seiner $\mathrm{F}$ ourier-Komponenten zusammensetzt, soll nun unmittelbar nachgeprüft werden: Dem Käferauge werden Muster geboten, die sich aus Komponenten bekannter Wirkung zusammensetzen. Die eben genannte These verlangt, daß sich die Wirkung des Musters dann additiv aus der Wirkung der Komponenten ergibt.

Dieses Experiment gibt Gelegenheit zur Lösung auch einer weiteren Frage: Überlagert man MusterKomponenten verschiedener Periodenlängen, so erhält man unterschiedliche Bilder bei verschiedener gegenseitiger Phasenlage. Durch Darbieten von Mustern aus gleichen periodischen Komponenten, aber unterschiedlicher Phasenlage, muß sich entscheiden lassen, ob das Käferauge für die Phasenlage empfindlich ist. Diese Frage wurde von Reichardi aufgeworfen, nachdem er die Bewegungsperzeption des Käferauges als Korrelations-Auswertung gedeutet hatte: War diese Deutung richtig, so durfte die Phasenlage auf die optokinetische Reaktion keinen Einfluß haben ${ }^{4}$.

Bei der Wahl der Muster folgte ich Ratschlägen von VARJú. Abb. 11 a und b zeigen die von ihm gewählten Musterkomponenten: Zwei Streifenmuster mit den Periodenlängen $22^{1} / 2^{\circ}$ und dem Vierfachen davon: $90^{\circ}$. Die $22^{1} / 2^{\circ}$-Komponente für sich allein muß nach dem in Abb. 10 veranschaulichten Effekt Bewegungswahrnehmungen in der Gegenrichtung zur Musterbewegung liefern (Konstante des Rasterzylinders $14^{\circ}$ ); die $90^{\circ}$-Komponente löst Reaktionen in der Bewegungsrichtung aus. Überlagert man
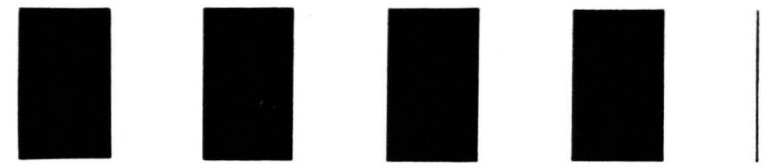

Abb. 11 a.

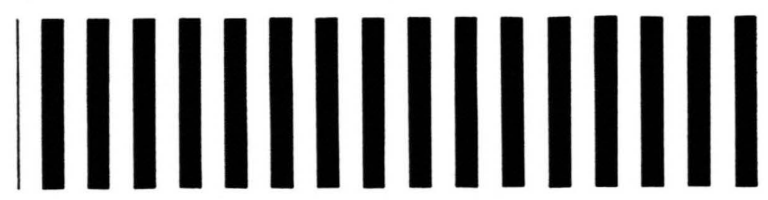

Abb. 11 b.

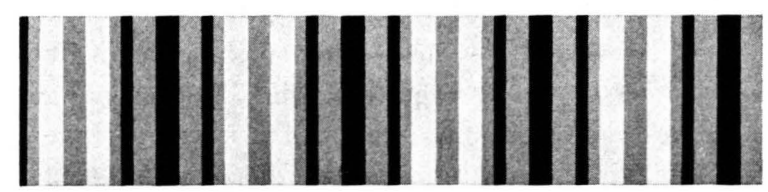

Abb. $11 \mathrm{c}$.

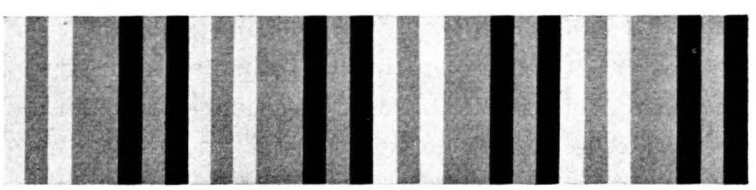

Abb. $11 \mathrm{~d}$.
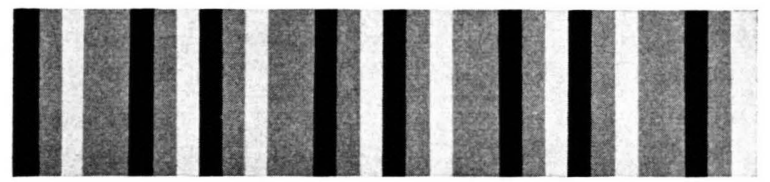

Abb. 11 e.

Abb. 11, von oben nach unten: a) Musterkomponente von $90^{\circ}$ Periodenlänge. b) Musterkomponente von $22^{1 / 2} \circ$ Periodenlänge. c) "Muster I“ und d) „Muster II", hervorgegangen aus der Überlagerung der beiden Komponenten, wobei (entsprechend der Mischregeln von Pigmentfarben) Schwarz+ Schwarz $=$ Schwarz; Wei + Wei $\beta=$ Wei $;$ und Schwarz + Wei $\beta=$ Grau ergibt. - Unterstes Muster $11 \mathrm{e}$, „Muster III“, hervorgegangen aus $11 \mathrm{~d}$ durch Vertauschen zweier Streifen pro Periode.

die beiden Muster so, daß jede Kontur des $90^{\circ}$. Musters mitten zwischen zwei Konturen des $22^{1} / 2^{\circ}$. Musters fällt, so entsteht das Muster Abb. 11 c. Fallen die Konturen beider Muster zusammen (um $180^{\circ}$ verschobene Phase der schmalstreifigen Komponente), so ergibt sich Abb. $11 \mathrm{~d}$.

Keiner der menschlichen Betrachter, denen ich die Muster $11 \mathrm{c}$ und $11 \mathrm{~d}$ ohne nähere Erklärung vorlegte, erkannte die Gemeinsamkeit ihrer Entstehung. Allen fielen jedoch die Unterschiede in die Augen: $11 \mathrm{c}$ ist in bezug auf die breiten Schwarzstreifen symmetrisch, $11 \mathrm{~d}$ hat keine solche Symmetrieachse; in $11 \mathrm{c}$ kommen $5^{5} / 8^{\circ}$ breite Schwarzstreifen vor, in $11 \mathrm{~d}$ stattdessen $22^{1} / 2^{\circ}$ breite Grausteifen; in $11 \mathrm{~d}$ 
kommt an je einer Kontur pro Periode ein SchwarzWeiß-Kontrast vor, in $11 \mathrm{c}$ nicht. Das menschliche Auge beurteilt ein Muster also nach anderen Prinzipien als den in ihm enthaltenen Fourier-Komponenten.

Die periodischen Komponenten Abb. 11 a und $\mathrm{b}$ sind Streifenmuster mit scharfen Konturen; sie sind also keine reinen Fourier-Komponenten, sondern enthalten selbst mehr als eine - im Prinzip unendlich viele - F o u ri e r - Komponenten. Da sich aber überall die Summenwirkung der Komponenten bestätigt, ändert es nichts an dem Grundgedanken des Versuchs, wenn die Komponenten selbst zusammengesetzt sind.

Um das vermutete Auswertungsprinzip des Käfers im Unterschied zur Sehweise des Menschenauges noch besonders drastisch zum Ausdruck zu bringen, bat ich VARJÚ um ein drittes zusammengesetztes Muster: Dieses sollte für das menschliche Auge von Abb. $11 \mathrm{c}$ oder d so wenig als möglich unterschieden sein, aber - zugeschnitten auf das Käferauge einen anderen Gehalt an periodischen Komponenten besitzen. Die Wahl fiel auf Muster $11 \mathrm{e}$, das durch bloße Vertauschung zweier Streifen pro Periodizitätsintervall aus $11 \mathrm{~d}$ entstanden ist. $11 \mathrm{~d}$ und $11 \mathrm{e}$ gleichen einander somit in Konturenanzahl, Streifenbreite und Helligkeitsabweichung vom Mittelwert, während $11 \mathrm{c}$ und $11 \mathrm{~d}$ die gleichen F o u ri e r-Komponenten besitzen. Der Versuch mit den Mustern 11 c bis 11 e war somit ein mehrfacher Test für die Bewegungsauswertung des Käferauges.

Abb. 12 zeigt das Versuchsergebnis. Daß alle Kurven tatsächlich als Summen der Effekte der F o u ri er-Komponenten ohne Berücksichtigung der Phasenbeziehung zu beschreiben sind, ist von VARJú quantitativ bearbeitet worden und wird von ihm berichtet ${ }^{5}$. Qualitativ läßt die Abbildung aber bereits folgendes erkennen: Die beiden ersten, für die menschliche Sehweise sehr verschiedenartigen Muster lösten im Rahmen der Meßgenauigkeit die gleichen Reaktionen aus. Ein Einfluß der Phasenbeziehungen zwischen den Musterkomponenten ist also nicht zu erkennen. Die Kurven schneiden die Null-Linie mit positiver Steigung, ein Zeichen dafür, daß im Einklang mit Abb. 9 die Wirkung der kleinen Periodenlänge bei geringeren, die der großen bei höheren Geschwindigkeiten überwiegt; die kleine Periodenlänge war, wie oben gesagt, so bemessen, daß sie Gegenreaktionen auslösen mußte (Abb. 10).

Einen völlig anderen Verlauf hat die Reaktionskurve auf das dritte zusammengesetzte Muster: Sie hat ein Maximum gerade an der Nullstelle der Kur- ven I und II; die Amplitude ist dort etwa doppelt so hoch wie deren Maximalamplitude. Nirgends setzt sich der Einfluß der $22^{1} / 2^{\circ}$-Periode soweit durch, daß negative Reaktionen auftreten. - Diese Unterschiede zu den anderen Kurven können nur dadurch zustandekommen, daß durch die Vertauschung der beiden Streifen neue F o u ri e r - Komponenten auf-

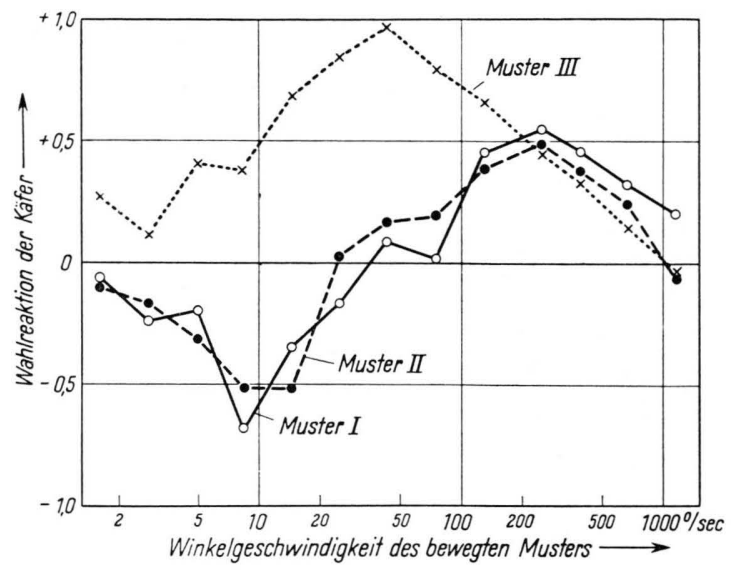

Abb. 12. Reaktionen der Käfer auf die Bewegung von Muster I, II und III. Da es unmöglich ist, sämtliche 39 Meßwerte aller 3 Kurven in einem durchlaufenden Experiment an ein und demselben Käfer zu gewinnen, wurde der Versuch in zwei Meßserien aufgeteilt: An 2 Käfern wurden die Winkelgeschwindigkeiten 1,$6 ; 4,8 ; 14,4 \ldots \%$ sec und an 2 anderen die dazwischen liegenden Winkelgeschwindigkeiten 2,75; 8,$25 ; 24,7 \ldots \%$ sec usw., jeweils mit allen 3 Mustern, geprüft. $\mathrm{Um}$ die individuellen Reaktionsstärke-Unterschiede der 4 Käfer nicht im Kurvenbild erscheinen zu lassen, wurden die Gesamtkurven der 4 Käfer mit Normierungsfaktoren $(0,9$; $1,1 ; 1,1 ; 1,5)$ multipliziert. Aufgezeichnet wurden die Durchschnittswerte zwischen den so normierten Einzelwerten.

getreten und stark in den Vordergrund getreten sind. Wie die mathematische Betrachtung zeigte ${ }^{5}$, handelt es sich dabei im wesentlichen um eine $45^{\circ}$-Komponente; das Maximum ihrer Wirkung muß also zwischen den Maxima der $22^{1} / 2^{\circ}$-Komponente und $90^{\circ}$. Komponente liegen, was die Abbildung bestätigt. Beim Blick auf Abb. 11 e kann auch der Mensch mit gutem Willen diese $45^{\circ}$-Periode erahnen; doch ist ihr Überwiegen anschaulich nicht wahrzunehmen. Die Auswertung periodischer Muster erfolgt eben bei uns nicht im Sinne einer F o u ri e r - Analyse. Beim Käferauge ist dies jedoch im Effekt der Fall.

Dieses Experiment bestätigt also in vollem Umfang die zuerst von Reichardt und Varju ${ }^{4,5}$ theoretisch hergeleitete Aussage: Die optokinetische Wirksamkeit bewegter periodischer Muster ergibt sich additiv aus der Wirksamkeit der in ihnen enthaltenen F o u ri er-Komponenten. Damit ist die erste der 
drei Fragen, die in der Einleitung gestellt wurden, beantwortet.

Damit ist auch die Frage gelöst, ob es die bewegten Konturen, für das Auge also Helligkeitssprünge (Änderungen!) oder ob es die Helligkeitswerte selbst sind, die den Informationsgehalt der Bewegung an das Käferauge vermitteln. Das Versuchsergebnis Abb. 3 schien für die erste, Abb. 6 für die zweite Alternative zu sprechen. Die Frage offenbart sich nun als unauflösbare Scheinfrage; sie enthält eine unrichtige Alternative. Entscheidend sind die Fou ri er-Komponenten, also sinusförmige Änderungen der Helligkeit; in ihnen sind bekanntlich Steigungen und Absolutwerte stets streng miteinander verknüpft und auf keine Weise unabhängig voneinandər zu behandeln.

\section{Multiplikation zwischen den} Meldungen ben a chbarter Sehelemente

$\mathrm{Da}$ die Helligkeitsmeldungen benachbarter Sehelemente beim Bewegungssehen ihrem Betrag nach miteinander multipliziert werden und nach diesem Formalismus die Intensität der Seitentendenzen beeinflussen, hatte ich bisher ${ }^{3}$ aus der allgemeinen Form der in bestimmten Versuchen entstehenden Reaktionskurven abgeleitet. Die damaligen Kurven erlaubten jedoch keine quantitativen Aussagen, und zwar, wie ich jetzt weiß, aus zwei Gründen: Wegen der Mitverwertung von Meßkurven, die den Wert 1 erreichten, also in den nichtlinearen Bereich der Meßmethodik hineinragten, und wegen der Verwendung von $\mathrm{O}$ stwalds Graustufen. Diese bilden keine arithmetische, sondern eine an die menschliche subjektive Grauskala angeglichene geometrische Reihe. Für das Käferauge waren die Ostwaldschen Graustufen offensichtlich nicht gleichabständig, wie sich an Asymmetrien in den damaligen Meßkurven zeigte. - Jetzt konnte ich mit genauerer quantitativer Methodik und den in Versuch 1 bewährten Graupapieren darangehen, die Multiplikation zwischen den Meldungen zusammenwirkender Ommatidien nachzuprüfen.

Das Prinzip der Reizanordnung ist auf Abb. 13 skizziert: Grauflächen bewegen sich vor einem Schwarz-Weiß-Hintergrund und rufen dadurch in aufeinanderfolgenden Positionen abwechselnd ein Hellerwerden (beim Bedecken schwarzer Hintergrundsstreifen) und ein Dunklerwerden (beim Bedecken weißer Hintergrundsstreifen) hervor. Zwi- schen der skizzierten Anordnung und dem Käferauge befindet sich im Versuch noch der Rasterzylinder mit senkrechten Schlitzen im Winkelabstand von $14^{\circ}$. Da die schwarzen und weißen Streifen am Hinter-

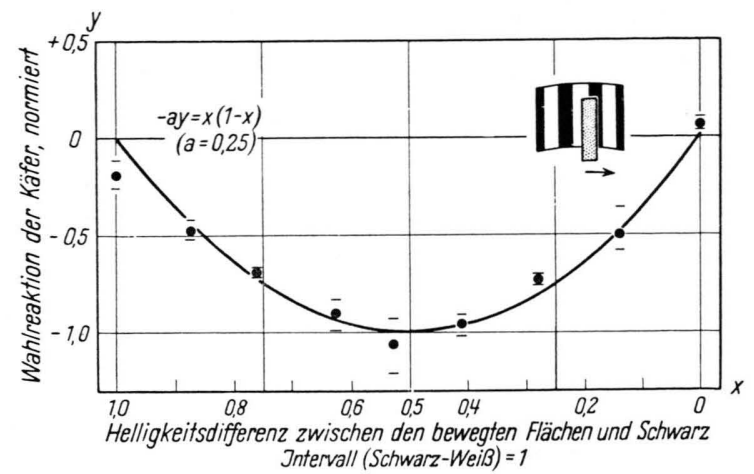

Abb. 13. Reaktionen der Käfer auf die Bewegung grauer Flächen vor schwarz-weißem Hintergrund. Der Käfer sah die angedeutete Musteranordnung durch einen Rasterzylinder, dessen schmale Schlitze den gleichen Winkelabstand voneinander hatten wie die Streifen des Hintergrundzylinders. Winkelgeschwindigkeit- der Grauflächen $74,1^{\circ}$ sec. Durchschnittswerte von 3 Käfern, die besonders gleichmäßig reagierten. Extreme Meßwerte durch waagerechte Striche angegeben. Eine weitere Messung wegen zu großer Streuung nicht verwertet. Die Formel gilt für die durchgezogene theoretische Kurve. Ihr Extremwert wurde gleich 1 gesetzt und die Mittelwertskurve so normiert, daß die Abweichungen von der theoretischen Kurve möglichst klein wurden.

grundszylinder $14^{\circ}$ breit waren, hatten aufeinanderfolgende Schlitze, vom Zentrum der Zylinder her gesehen, abwechselnd einen weißen und einen schwarzen Hintergrund. Der Sinn dieser Anordnung war der: Die bewegten Flächen sollten je nach ihrer Helligkeit (Graustufe) verschieden große Helligkeitssprünge erzeugen; dabei sollte aber die Summe der Reize, die in je zwei zusammenwirkende Ommatidien fielen, immer gleich sein, und zwar gleich dem Sprung zwischen Schwarz und Weiß. (Bezeichnet man den Sprung vom jeweiligen Grau zu Weiß als $x$ und setzt den Sprung von Schwarz zu Weiß = 1, so sind die Beträge der jeweils zusammenwirkenden Reize $x$ und $1-x$; ihre Summe ist also $=1$ ). Hierdurch wird die Reaktionskurve bei der Variation des Grauwertes der rotierenden Flächen zu einem empfindlichen Indikator für den Formalismus der Wechselwirkung zwischen den Meldungen der Ommatidien. Im Falle additiven Zusammenwirkens muß die Reaktion $y=x+(1-x)=1$, also unabhängig von einer Variation von $x$ sein, während sich z. B. bei multiplikativer Wechselwirkung die Parabel

$$
y=x(1-x)
$$

ergeben muß. 
Um die Art der Wechselwirkung möglichst rein in den Reaktionskurven zum Ausdruck kommen zu lassen, ergriff ich Maßnahmen, um eventuelle Nichtlinearitäten in der Zuleitung von den Sinnesorganen her von vorneherein abzuschwächen oder zu unterdrücken. Mit Hilfe möglichst schmaler Schlitze verengte ich die Durchblicke auf Hintergrundszylinder und rotierende Grauflächen und hielt dadurch alle Helligkeitsänderungen, die die Ommatidien trafen, so klein als möglich. Dies mußte für den Fall einer nichtlinearen Übersetzung linearisierend wirken; denn je kleiner ein Abschnitt aus einer gekrümmten Kennlinie, desto mehr ähnelt er, als Ganzes betrachtet, einer Geraden. Ferner wählte ich eine möglichst hohe Geschwindigkeit; denn falls eine nichtlineare Dynamik, z. B. eine Adaptation im Spiel war, wurde ihr dadurch weniger Zeit gelassen, sich auszuwirken. - Um die Beleuchtung von Hintergrund und bewegten Grauflächen möglichst gleich zu machen, rotierten die Grauflächen im Unterschied zu früher ganz dicht $(4 \mathrm{~mm})$ vor dem Schwarz-Weiß-Muster.

Abb. 13 zeigt das Versuchsergebnis. Die Meßpunkte liegen dicht an der Parabel $-0,25 y$ $=x(1-x)$ und bestätigen damit, daß die Wechselwirkung zwischen den Meldungen benachbarter Ommatidien einer Multiplikation gleicht oder ihr sehr nahekommt. Die verbesserte Methodik hat also die bisherigen Aussagen quantitativ bestätigt.

$\mathrm{Da}$ die Kurve im Bereich negativer Reaktionen liegt, ist die Folge davon, daß jeweils ein +-Reiz (Hellerwerden) und ein --Reiz (Dunklerwerden) zusammenwirken. Wie früher ${ }^{3}$ genauer beschrieben, bringen Reizfolgen aus zwei +-Reizen oder aus zwei - -Reizen optokinetische Reaktionen in der Bewegungsrichtung hervor, während Reizfolgen aus zwei Reizen entgegengesetzten Vorzeichens entgegengesetzte Reaktionen auslösen. Dieses Verhalten entspricht der Vorzeichenregel der Multiplikation.

Von einer Asymmetrie in der Verteilung der Meßpunkte ist nichts zu bemerken; Nichtlinearitäten in den Zuleitungen zur Multiplikations-Instanz sind also nicht vorhanden oder wurden durch die beschriebenen Maßnahmen in den Bereich der Meßgenauigkeit herabgedrückt.

\section{Nichtlinearitäten in den}

Zuleitungen zur Multiplikations instanz

Im vorigen Versuch hatte ich eventuelle Nichtlinearitäten in der Reizverarbeitung von vornherein abzuschwächen versucht, um den Prozeß der Wechselwirkung zwischen den Sinnesmeldungen möglichst rein zum Ausdruck zu bringen. Durch das nun folgende Experiment wollte ich erfahren, ob die Übertragung der Sinnesmeldungen überhaupt Nicht- linearitäten enthält (falls nein, wäre die Vorsicht im vorigen Versuch unnötig gewesen). Im Unterschied zum vorigen Versuch wählte ich nun möglichst hohe Amplituden der Helligkeitswechsel für die Ommatidien der Käferaugen: Ich beschränkte deren Gesichtsfeld gar nicht, verwendete also keinen Rasterzylinder. Ferner wählte ich möglichst geringe Bewegungs-Geschwindigkeiten der Grauflächen, im Extremfall $2,3^{\circ} / \mathrm{sec}$.

Wegen des Fehlens des Rasterzylinders wurde jetzt nicht wie im vorigen Versuch nur jedes zweite Sehelement von Helligkeitsänderungs-Reizen getroffen, sondern jedes unmittelbar benachbarte. Dadurch mußte sich die Form der Reaktionskurve ändern: Wegen der Breite des Hintergrundsstreifens $\left(14^{\circ}\right)$ empfingen nun in vielen Fällen benachbarte Ommatidien (rund $7^{\circ}$ Winkelabstand zwischen den Achsen) die gleiche Grundhelligkeit und wurden darum bei der Bewegung der Grauflächen im gleichen Helligkeitsänderungs-Sinn gereizt. Damit entstand eine positive Reaktionskomponente, ein Anteil an Mit-Tendenz. Das war kein Nachteil, sondern bot für den gegenwärtigen Zweck einen Vorteil: Jetzt endete die Reaktionskurve nicht beiderseits an der NullLinie, sondern bei positiven Reaktionswerten; sie schnitt also die Null-Linie. Außerdem wurden die Anstiege der Kurve steiler und die Krümmung beim Minimum schärfer; das liegt daran, daß die positive Komponente die Form $x^{2}+(1-x)^{2}$ besitzt und sich zu der in Abb. 13 dargestellten Kurve $-x(1-x)$ hinzuaddiert (aufgetragene Kurven eines ähnlichen mathematischen Ausdrucks s. l. c. ${ }^{3}$ ). All diese Merkmale sind dazu geeignet, eine etwaige Asymmetrie der Reaktionskurve im Verhältnis zur Abszisse deutlicher zum Ausdruck kommen zu lassen, als das bei der Kurve Abb. 13 möglich gewesen wäre.

Die durchgezogene Kurve der Abb. 14 zeigt die Reaktionen eines Käfers unter den angegebenen, zum Herauslocken etwaiger Nichtlinearitäten bestmöglichen Bedingungen. Wie man sieht, ist sie tatsächlich asymmetrisch: Ihr Tiefstwert liegt nicht beim Abszissenwert 0,5, sondern ist deutlich in Richtung zu dunkleren Grauwerten verschoben.

Als Gegensatz zu der asymmetrischen Reaktionskurve ist in Abb. 14 eine so gut wie symmetrische Meßkurve eingetragen (unterbrochen gezeichnet). Sie ist unter bestmöglich linearisierenden Bedingungen aufgenommen worden: Bei hoher Mustergeschwindigkeit und unter Verwendung eines Rasterzylinders. Die Rasteröffnungen waren $7^{\circ}$ voneinander entfernt, die SchwarzWeiß-Hintergrundsstreifen waren $7^{\circ}$ breit; darum ergab sich eine etwas andere, weniger spitze Kurvenform - jedoch die der Form der asymmetrischen Kurve ähnlichste, die unter Verwendung eines Rasterzylinders erreichbar ist. Die Asymmetrie der durchgezogenen Kurve tritt nun im Vergleich besonders deutlich hervor: Sie geht so weit, daß zwei ihrer Meßpunkte im Positiven liegen, die bei der Vergleichskurve negativ sind - dar- 


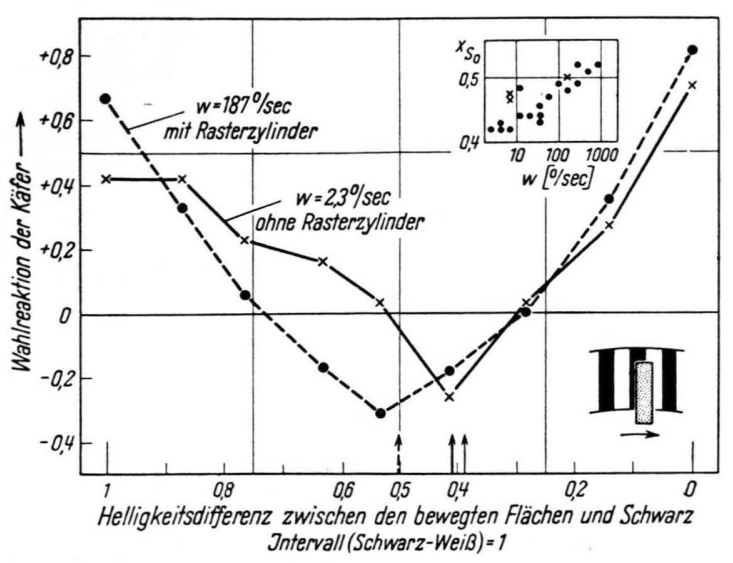

Abb. 14. Erklärung der Kurven im Text. Die beiden dicken senkrechten Pfeile am Unterrand der Abbildung markieren den Abszissenwert $X_{\mathrm{S}_{0}}$ in der Mitte zwischen den Nulldurchgängen der beiden Kurven. Der dünne senkrechte Pfeil markiert den vermutlichen Abszissenwert des Tiefpunktes der Reaktionskurve, falls diese in ihrem gesamten Verlauf die gleiche Asymmetrie zeigt, wie sie in der Lage der Nulldurchgänge zum Ausdruck kommt. - Die Einschaltfigur zeigt die in Einzelmessungen gewonnenen $X_{\mathrm{S}_{0}}$-Werte in Abhängigkeit von der Winkelgeschwindigkeit $w$ der Grauflächen. Punkte: Versuche ohne Rasterzylinder; liegende Kreuze: Versuche mit Rasterzylinder.

unter der Meßpunkt bei dem am besten angenäherten Mittelgrau.

Damit war eine Nichtlinearität in der Reizübertragung im Käferauge nachgewiesen. Um festzustellen, ob sie auf einer nichtlinearen Kennlinie * oder auf einer nichtlinearen Kinetik * beruhte, variierte ich in dem beschriebenen Versuch die Mustergeschwindigkeit. Das Ergebnis war: Die Asymmetrie verlor sich mit wachsender Grauflächen-Geschwindigkeit. Als ungefähre Maßzahl für die Asymmetrie der entstehenden Einzelkurven wählte ich den Abszissenwert in der Mitte zwischen den Nulldurchgängen der Reaktionskurve; die Pfeile auf Abb. 14 markieren diese Werte für die dort aufgezeichneten beiden Reaktionskurven. Die Einschaltfigur in Abb. 14 zeigt diese Positionen für alle angestellten Messungen, aufgetragen gegen die jeweilige GrauflächenGeschwindigkeit. Wie die Meßpunkte deutlich zeigen, ist die gefundene Nichtlinearität Geschwindigkeits-abhängig. Sie muß also auf einem dynamischen Vorgang beruhen, während die Informationsübertra-

* Eine nichtlineare Kennlinie charakterisiert einen Mechanismus, der die Augenblicks-Werte der Eingangsgröße nach einem nichtlinearen Schlüssel in die Werte der Ausgangsgröße übersetzt. Ein nichtlinearer Zusammenhang zwischen Eingang und Ausgang kann jedoch auch dadurch zustandekommen, daß sich beim Vorgang der Transformation die gung zwischen Reizaufnahme und Multiplikationsinstanz - und damit wahrscheinlich ${ }^{* *}$ auch die Helligkeitsübersetzung im Sinneselement - linear sein muß. Da es sich bei der damit festgestellten dynamischen Nichtlinearität um die Änderung in der Reizbeantwortung von Sinneselementen handelt, fällt dieser Vorgang unter den biologischen Oberbegriff der Adaptation.

Mit diesem Begriff steht im Einklang, daß sich der im Experiment bestimmte physiologische Mittelwert zwischen den Extremen Schwarz und Weiß mit abnehmender Reizfrequenz in Richtung zu Schwarz, also skalenabwärts zu absolut kleineren Reizen hin verschiebt. Es sind zwei Kennzeichen jedes Adaptationsvorgangs, zum einen, daß er, wenn er einem sich ändernden Reiz schnell genug folgen kann, diesen Reiz in seiner Wirkung abschwächt; und zum anderen, daß er einem größer werdenden Reiz schneller folgt als einem abnehmenden (z. B. dauert die Dunkeladaptation von einem bestimmten HelladaptationsZustand aus um ein Vielfaches länger, als der entsprechende Vorgang in umgekehrter Richtung). Das bedeutet, wie ich vermute, für das Käferauge: Verändern sich die Helligkeiten für die Ommatidien wegen geringer Geschwindigkeit der rotierenden Flächen langsam, so vermag die Adaptation den Helligkeitsänderungen teilweise zu folgen. Dadurch müssen die Hellreize stärker geschwächt werden (durch die Helladaptation) als die Dunkelreize durch die langsamer verlaufende Dunkeladaptation. Infolgedessen muß der physiologische Helligkeits-Mittelwert zwischen den stärker geschwächten Hellreiz-Wirkungen und den weniger geschwächten Dunkelreiz-Wirkungen - durch die Adaptation absinken.

Der beschriebene Versuch gibt also Auskunft über Empfindlichkeitsänderung der Ommatidien. Dies geschieht jedoch auf einem sehr mittelbaren Wege. Daher plane ich mit dieser Methode keine weitere Analyse der Adaptation des Chlorophanus-Auges. Doch möchte ich ein Maß für die gefundene nichtlineare Verzerrung der Helligkeits-Übersetzung angeben, das einen ungefähren Vergleich mit der Grauskala des Menschenauges gestattet, und wähle die relative Verschiebung des Mittelwertes gegen eines der Skalenenden. Setzt man einen linearen Skalenabschnitt gleich 1, so liegt sein Mittelwert bei 0,5 . Gehen wir von der arithmetischen Grauskala aus, so ist ihr Mittelwert für das menschliche Auge um etwa

Nachwirkungen zeitlich vorausgegangener Werte mit auswirken, und dies in nichtlinearer Weise geschieht. Im letzteren Fall handelt es sich dann um eine nichtlineare Kinetik.

* Zu der Frage, warum dieser Schluß nicht unbedingt zwingend ist, s. l. c. ${ }^{4}$ 
0,26 auf 0,24 verschoben. Betrachtet man die größte mögliche Verschiebung $(0,5)$ als Einheit, so ist die Verzerrung der Grauskala für das Menschenauge durch den Wert $0,26 / 0,5=0,52$ oder $52 \%$ charakterisiert. Für die Reaktion des Käfers als Ganzes liegt der Mittelwert im Extremfall etwa bei Grau 0,4 (s. Legende zu Abb. 13), was ein Verzerrtsein der Grauskala um 20\% bedeutet. Setzt man voraus, daß die Reaktionskurve aus der Multiplikation zwischen den Werten zweier Grauskalen entsteht, von denen hauptsächlich die eine (die von der Helladaptation betroffene) für die Verzerrung verantwortlich ist, so ergibt sich für diese ein etwas größerer Wert. Er dürfte etwa 25\% betragen. Das hiermit verwendete Maß für die Verzerrung einer linearen Skala kann nur einen ungefähren Hinweis ermöglichen. Beim Vorliegen genauerer Messungen werden andere Kriterien heranzuziehen sein.

Abschließend versuchte ich festzustellen, ob nur die Geschwindigkeit, oder ob auch die Verkleinerung des Gesichtsfeldes der Einzelommatidien durch den Rasterzylinder einen Einfluß auf die Verschiebung des „physiologischen Mittelgrau“ nimmt. Die Lage der Meßpunkte „x“ auf der Einschaltfigur sprechen dafür, daß dies der Fall ist. Durch den Rasterzylinder wird die offenbar werdende Nichtlinearität verringert.

Versuch 8 und 9 haben damit die Frage nach der physiologischen Grauskala des Käferauges in folgendem Sinne beantwortet: Bei der in allen Versuchen verwendeten Beleuchtungsstärke und bei Geschwindigkeiten oberhalb des Reaktionsoptimums übersetzt das Käferauge Helligkeitswerte im Rahmen der Meßgenauigkeit linear in physiologische Helligkeitsmeldungen * . Bei geringer werdenden Geschwindigkeiten macht sich eine Adaptation bemerkbar. Sie bringt eine nichtlineare Verzerrung der Grauskala hervor, die im Extrem wahrscheinlich bis zu 25\% geht. Sowohl höhere Geschwindigkeit als auch die Verwendung von Rasterzylindern verringern diese Nichtlinearität.

Für die theoretische Analyse der Bewegungsperzeption ist die Kenntnis wichtig, unter welchen Umständen und in welchem Umfang Nichtlinearitäten in den sonst linearen Reiz-Reaktionszusammenhang hineinwirken können: In erster Näherung ist es sinnvoll, mit der Voraussetzung der Linearität zu arbeiten; mit Abweichungen, die über den Rahmen der Meßgenauigkeit hinausgehen, braucht höchstens bei sehr geringen Geschwindigkeiten und großen Rasteröffnungen gerechnet zu werden.

\footnotetext{
* Nichtlinearitäten, die sich vor der nächsten Verrechnung durch einen Gegenvorgang wieder ausgleichen, sind damit natürlich nicht ausgeschlossen (s. B. Hassenstein u. W. ReIChardt, Z. Naturforschg. 8 b, 518 [1953]).
}

10. Muster aus statistisch abwechselnden Helligkeitswerten

Drei Gründe sprachen dafür, auch Muster aus statistisch abwechselnden Helligkeitswerten (Abb. 15 a, b) auf ihre optokinetische Wirksamkeit für das Käferauge zu untersuchen: 1. die optische Umwelt für Insektenaugen besteht in freier Natur aus periodisch und aus statistisch abwechselnden Helligkeitswerten; daher ist es wichtig, auch diese Komponente in ihrer Wirkung zu kennen. 2. Bewegungsperzeption nach dem Korrelationsprinzip heißt Auswerten statistischer Verhältnisse, nicht Verfolgen bewegter Musteranteile. Das Wesen der Bewegungsperzeption des Insektenauges muß sich daher am eindrucksvollsten dort offenbaren, wo ein Verfolgen bewegter Musteranteile am wenigsten in Frage kommt: Bei der Bewegung regellos zusammengesetzter Muster größtmöglicher Feinheit, wahrgenommen durch Rasteröffnungen möglichst weiten Abstands voneinander. 3. Die symmetrische glockenartige Form der Reaktionskurve auf Sinusmuster Abb. 5 ließ sich theoretisch nur durch die Annahme deuten, daß ein durch eine partielle Differentialgleichung zu beschreibender UUbertragungsvorgang in das Auswertesystem eingefügt ist ${ }^{4}$. Die mathematische Analyse ${ }^{4}$ förderte ein Merkmal zutage, das dieses Funktionsglied auch unmittelbar qualitativ nachzuweisen gestatten mußte: Ein Überschwingen der Reaktionskurve zu negativen Reaktionswerten bei sehr geringen Geschwindigkeiten eines idealen statistischen Helligkeitsmusters.

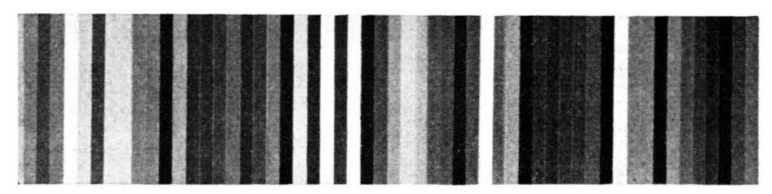

Abb. 15 a.

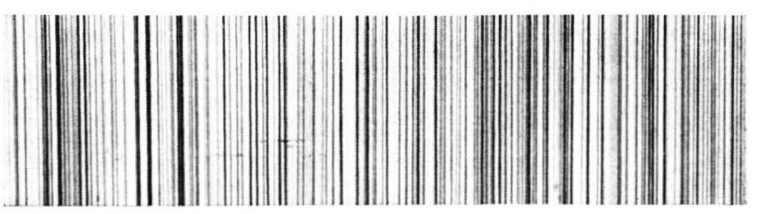

Abb. 15 b.

Abb. 15. Oben: Statistische Streifenmuster, verwendet in dem $1958{ }^{2}$ beschriebenen Versuch. Unten: Statistische Streifenmuster, verwendet in Versuch 10. Beide Muster auf photographischem Wege hergestellt.

Auf verschieden schnelle Bewegungen des Musters Abb. 15 a hatte ich seinerzeit ${ }^{2}$ Reaktionskurven erhalten, die bei keiner Geschwindigkeit negativ wurden. 
Daraufhin verwendete ich diesmal ein viel feineres Muster aus rund $1^{\circ}$ breiten Streifen (Abb. 15 b). Da die Reaktionen der Käfer auf die Bewegung dieses Musters zum Registrieren zu gering waren, mußte ich ausnahmsweise, wie in der Einleitung erwähnt, Rasterzylinder mit Schlitzen von $7^{\circ}$ Abstand verwenden. Dadurch summierten sich die Auswertungsergebnisse von benachbarten und übernächsten Ommatidien ${ }^{3}$.

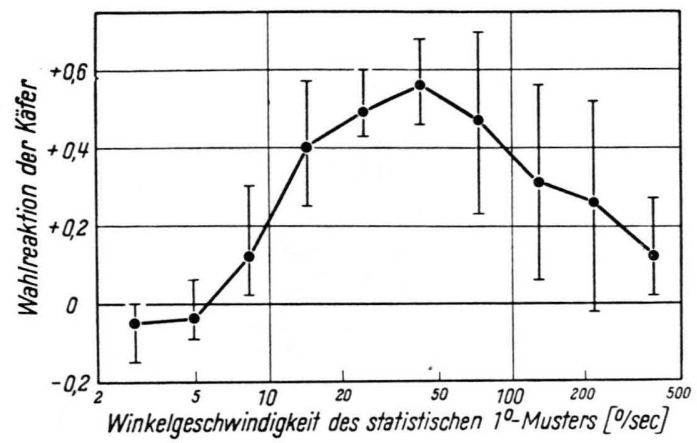

Abb. 16. Reaktionen auf die Bewegung des Musters Abb. 15 b. Durchschnittswerte von 5 Käfern. Eine weitere Messung wegen zu starker Streuung nicht verwertet. Extreme Meßwerte durch waagerechte Striche angegeben.

Abb. 16 zeigt die Versuchsergebnisse: Auf den ersten Blick sieht die Geschwindigkeits-Abhängigkeit der Reaktion auf den statistischen $1^{\circ}$-Zylinder ähnlich wie die von periodischen Mustern aus (Abb. 3, $5,7)$. Sie zeigt jedoch ein ganz aus dem Rahmen fallendes Merkmal: Bei der Winkelgeschwindigkeit von etwa $6^{\circ} / \mathrm{sec}$ ist die Reaktion gleich Null, und bei noch geringeren Geschwindigkeiten wird sie negativ. Die Käfer zeigen hier also eine Wendetendenz entgegen der tatsächlichen Zylinderdrehung. Der negative Kurvenanteil trat bei allen 7 untersuchten Käfern auf; der einzige Käfer, der bei der Winkelgeschwindigkeit $4,8^{\circ} / \mathrm{sec}$ eine positive Reaktion zeigte (oberer Extremwert bei dieser Geschwindigkeit auf Abb. 16), reagierte stark negativ bei $2,8^{\circ} / \mathrm{sec}$ (unterer Extremwert). Sämtliche 11 Einzelmessungen an den übrigen 6 Käfern lagen bei den zwei niedrigsten Geschwindigkeiten ohne Ausnahme im negativen Bereich. An der Zuverlässigkeit der Aussage ist also kein Zweifel.

Die Gegenreaktionen der Käfer auf das langsam rotierende statistische $1^{\circ}$-Muster lassen sich auf keine der beiden bisher beschriebenen Arten von Gegenreaktionen zurückführen; sie beruhen nicht auf geometrischen Interferenzen zwischen gegeneinander bewegten Rastern (s. Abb. 10) und nicht auf dem Wechsel der Vorzeichen der Reize in aufeinanderfolgenden Sehelementen (s. Abb. 13). Da die
Gegenreaktionen hier Geschwindigkeits-abhängig auftreten, müssen sie - wenn man ähnlich folgert wie im vorigen Versuch bei der Adaptation als „dynamischer Nichtlinearität" - die Folge eines dynamischen Vorgangs sein:

Eine gegen die Musterbewegung gerichtete Reaktion muß, sofern keine geometrischen Interferenzbewegungen (Abb. 10) im Spiel sind, auf der Multiplikation zwischen +- und - -Meldungen beruhen. Ein bewegtes Muster aus statistisch gemischten Helligkeitswerten, gibt aber bei der Abtastung durch zwei Rezeptoren gleichviele Kombinationen ++ , ,--+- und $-+{ }^{1,3}$. Durch träge Übertragung können die Komponenten ++ und -- nur hervorgehoben, nicht unterdrückt werden ${ }^{1,3}$. Ein Überwiegen der Kombinationen +- und -+ ist demgegenüber nur denkbar, wenn im Verlauf der Auswertung in jeweils einem der beiden abtastenden Rezeptoren +-Meldungen in --Meldungen oder --Meldungen in +-Meldungen umgewandelt werden. Wenn ein Übertragungsvorgang mit bestimmter Dynamik für solch eine Vorzeichenumkehr verantwortlich ist (Geschwindigkeits-Abhängigkeit des Vorzeichens der Reaktion!) so bedeutet es, daß sein Funktionszustand auf eine Auslenkung hin nicht auf direktem Wege die Ruhelage erreicht, sondern vorher nach der Gegenseite überschwingt. Gerade eine solche Übertragungseigenschaft hatten nun REICHARDT und VARJú bereits vorher aus einem ganz andersartigen Versuchsergebnis erschlossen, nämlich aus der Reaktionskurve auf das bewegte Sinusmuster (Abb. 5); sie hatten das negative Ende der Reaktionskurve Abb. 16 als neuen qualitativen Effekt vorausgesagt. - Anschaulich kann man folgende Deutung geben: Bei geringen Geschwindigkeiten wird die Nachwirkung jedes Einzelreizes gerade in dem überschwingenden Zustand von den Meldungen aus dem Nachbarommatidium getroffen, deren Vorzeichen nicht verändert sind. Das ergibt dann die außergewöhnlichen + - - und -+ -Kombinationen.

Die Reaktionskurve Abb. 16 steigt gemäß der theoretischen Voraussage ${ }^{4}$ zuerst steil und dann flacher zu ihrem Maximum an. Sie fällt dann aber schon von $45^{\circ} / \mathrm{sec}$ an, also viel früher, als für den Idealfall des statistischen Zylinders vorausberechnet ${ }^{4}$, mit geringerer Steigung wieder ab. Auf dem absteigenden Schenkel wird die Streuung der Meßpunkte sehr groß. Die beiden letzten Befunde brachten uns auf den Gedanken, daß jedes auf einen Zylinder aufgebrachte, also in sich zurücklaufende Streifenmuster in bezug auf die langwelligen F o u r i e r - Komponenten höchst unvollkommen ist. 
Eine ideale eindimensionale statistische Verteilung enthält als Fourier-Komponente sämtliche Wellenlängen von 0 bis $\infty$ mit gleicher Amplitude. Ein in sich zurücklaufendes Muster auf einem Zylinder besitzt aber eine Komponente mit der größten Wellenlänge von $\lambda=360^{\circ}$, die nächstkleineren sind $180^{\circ}, 120^{\circ}, 90^{\circ}, 72^{\circ}$ usf., und erst bei noch kleineren Werten tritt eine dichtere Folge von Wellenlängen auf. Nur dort, wo die kleineren Wellenlängen für den Hauptanteil der Reaktion verantwortlich sind, ist daher eine Annäherung an die theoretische Kurve des Idealfalles des bewegten statistischen Helligkeitsmusters zu erwarten. Dies betrifft das Gebiet geringer Geschwindigkeiten, denn wie in Abschnitt 6 a dargelegt ist, liegen die Wirkungsmaxima kurzwelliger Sinuskomponenten bei kleineren Geschwindigkeiten; damit wird der Bereich der negativen Reaktionen zuverlässig wiedergegeben.

Aber auch im Bereich kurzer Wellenlängen weichen die verwendeten Muster Abb. 15 vom idealen statistischen Muster ab; sie besitzen keine unendlich feine, sondern eine recht grobe Struktur, enthalten also von einem Optimalwert an die kleineren Wellenlängen in abnehmendem Maße. Dieser Mangel ist bei dem breitstreifigen Muster 15 a natürlich viel größer als bei dem feiner gegliederten von $15 \mathrm{~b}$; daher kommt es vermutlich, daß durch langsame Geschwindigkeiten von Muster 15 a keine negativen Reaktionen ausgelöst wurden (s. $1958^{1}$, Abb. 4 und vgl. mit Abb. 4 der Arbeit von Reichardt und $\mathrm{V}_{\text {ARJ }}{ }^{4}$ ).
Damit ist die dritte in der Einleitung gestellte Frage in folgendem Sinne beantwortet: Mit einem Streifenzylinder läßt sich die Situation des bewegten idealen statistischen Musters nur unvollkommen verwirklichen. In demjenigen Geschwindigkeitsbereich, der die statistischen Züge des experimentell dargebotenen Musters am deutlichsten in der Reaktion zum Ausdruck kommen läßt, liegt aber zum Glück der interessanteste Teil der Reaktionskurve: Ein Bereich negativer optokinetischer Wendetendenz. Er läßt unmittelbar auf die Wirksamkeit einer übertragenden Instanz bestimmter Charakteristik ${ }^{4}$ schließen, deren Funktionswert auf eine einseitige Auslenkung hin vorübergehend über die Ruhelage hinaus zur Gegenseite überschwingt.

Meiner Frau danke ich für das Sammeln und Versenden der Versuchstiere, Herrn stud. Dietmar Todt für freiwillige wissenschaftliche Mitarbeit, Fräulein Angela Herrmann und Fräulein Ulrike Schönfeld für gewissenhafte und interessierte technische Mitarbeit, Herrn cand. Peter Kunze für kritische Durchsicht des Manuskripts, und Dr. Werner Reichardt und Dr. Dezsö Varjú, ohne deren theoretische Untersuchungen diese Arbeit nicht zustandegekommen wäre, für entscheidende Anregungen und hilfreiche Kritik.

\title{
Übertragungseigenschaften im Auswertesystem für das Bewegungssehen
}

(Folgerungen aus Experimenten an dem Rüsselkäfer Clorophanus viridis)

\author{
Von Werner Reichardt und Dezsö Varjé
}

Aus dem Max-Planck-Institut für Biologie in Tübingen, Forschungsgruppe Kybernetik

(Z. Naturforschg. 14 b, $674-689$ [1959]; eingegangen am 15. Mai 1959)

\begin{abstract}
Optokinetic reactions of the beetle Chlorophanus are not only elicited by moving figures distinguishable against their backgrounds, but also from movements of randomly constructed patterns of shades from white through black (random functions). The evaluation of relative motions by the central nervous system is made in accordance with the correlation principle ${ }^{1}$. The functional structure of perception, discussed in an earlier paper ${ }^{1}$, describes the reactions to motions of random functions but does not explain the strength of optokinetic reactions which are released by moving sinusoidal patterns. In the following paper the frequency response functions of the filters are determined in such a way that the functional structure (Fig. 1) describes the reactions to both sinusoidal and randomly constructed light patterns (Fig. 3 and 4). Two results follow from the analysis: 1 . The time constants of the low pass filters are $\tau_{\mathrm{F}}=1,6 \mathrm{sec}, \tau_{\mathrm{H}}=0,03 \mathrm{sec}$. 2 . The input filters cannot be of the differentiating type. Their frequency response function is proportional to the root of frequency. The kinetics of the low pass filters can be explained on the basis of a first order linear differential equation which corresponds to a monomolecular chemical reaction. The frequency response functions of the input filters can only be explained by a partial differential equation describing processes of diffusion.
\end{abstract}

In einer früheren Arbeit ${ }^{1}$ wurde auf der Basis der Funktionsstruktur für das Bewegungssehen des Rüsselkäfers Chlorophanus ${ }^{2,3}$ vorausgesagt, daß optomotorische Reaktionen nicht nur bei der Bewegung von Figuren, sondern auch bei der Bewegung von solchen Hellig. keitsmustern auftreten müßten, innerhalb deren die vorkommenden Helligkeitswerte statistisch voneinander un- abhängig sind (Helligkeits-Rauschfunktionen). Die Versuche ${ }^{4}$ haben diese Voraussage bestätigt.

1 W. Reichardt, Z. Naturforschg. 12 b, 448 [1957].

2 B. Hassenstein u. W. Reichardt, Z. Naturforschg. 11 b, 513 [1956].

3 B. Hassenstein, Z. vergleich. Physiol. 40, 556 [1958].

4 B. Hassenstein, Z. Naturforschg. 13 b, 1 [1958]. 